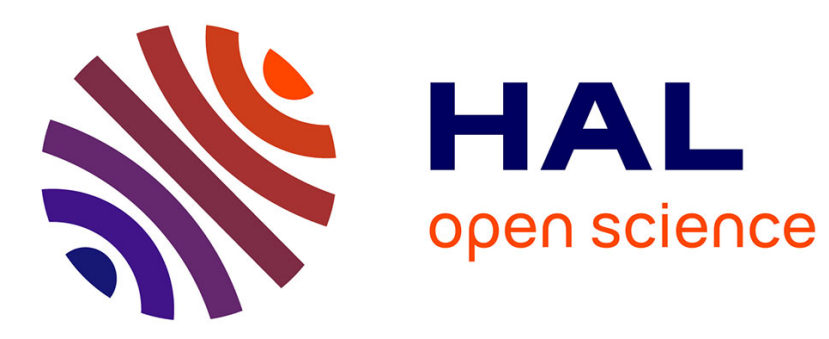

\title{
Capteurs acousto-électriques radiofréquences - Modes d'interrogation
}

\author{
Jean-Michel Friedt, Sylvain Ballandras
}

\section{To cite this version:}

Jean-Michel Friedt, Sylvain Ballandras. Capteurs acousto-électriques radiofréquences - Modes d'interrogation. Techniques de l'Ingenieur, 2020, E3212, pp.24. hal-03360774

\section{HAL Id: hal-03360774 \\ https://hal.science/hal-03360774}

Submitted on 1 Oct 2021

HAL is a multi-disciplinary open access archive for the deposit and dissemination of scientific research documents, whether they are published or not. The documents may come from teaching and research institutions in France or abroad, or from public or private research centers.
L'archive ouverte pluridisciplinaire HAL, est destinée au dépôt et à la diffusion de documents scientifiques de niveau recherche, publiés ou non, émanant des établissements d'enseignement et de recherche français ou étrangers, des laboratoires publics ou privés. 


\section{E3212 : CAPTEURS ACOUSTOÉLECTRIQUES RADIOFRÉQUENCES - MODES D'INTERROGATION \\ RADIOFREQUENCY ACOUSTIC PASSIVE WIRELESS SENSORS - INTERROGATIONS STRATEGIES}

\author{
J.-M Friedt \\ enseignant-chercheur \\ FEMTO-ST Time \& Frequency, Besançon, France \\ Email : jmfriedt@femto-st.fr
}

\author{
S. Ballandras \\ directeur général
}

frec $|n|$ sys, Besançon, France

Email : sylvain.ballandras@frecnsys.com

\begin{abstract}
Résumé-Les dispositifs à ondes élastiques opèrent comme capteurs grâce à la sensibilité de leurs caractéristiques physiques, notamment vitesse de l'onde, aux paramètres environnementaux. Ils sont exploités comme cible coopérative de RADAR à courte portée $(<\mathbf{1 0} \mathbf{~ m})$ en tant que capteurs passifs interrogeables sans fil. Une approche système nécessite de maîtriser la chaîne de mesure : conception de la cible pour retarder le signal au-delà du fond incohérent ; conception du capteur par choix du substrat piézoélectrique pour l'analyse de la fréquence de résonance ou phase du signal retardé ; mise en ouvre du RADAR et des algorithmes de traitements.

Acoustic wave transducers are considered as short range RADAR cooperative targets for probing through a radiofrequency link passive sensor responses. A systems engineering approach is needed to consistently address the whole measurement chain as described in this article : target design in order to delay the sensor response beyond clutter; physical quantity measurement through a resonance frequency or echo delay introduced by the sensing mechanism; short range RADAR implementation and associated signal processing schemes; and finally sensor design including a clever selection of the piezoelectric substrate. Application examples are given at the end of the presentation.
\end{abstract}

Mots clés : onde élastique de surface, RADAR courte portée, cible coopérative, capteur, High Overtone Bulk Acoustic Resonator

Keywords : surface acoustic wave, short range RADAR, cooperative target, sensor, High Overtone Bulk Acoustic Resonator

\section{TABLE DES MATIÈRES}

\section{$1 \quad$ Principe de mesure}

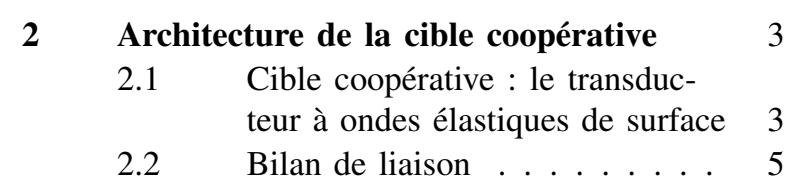

$3 \quad$ Électronique de mesure

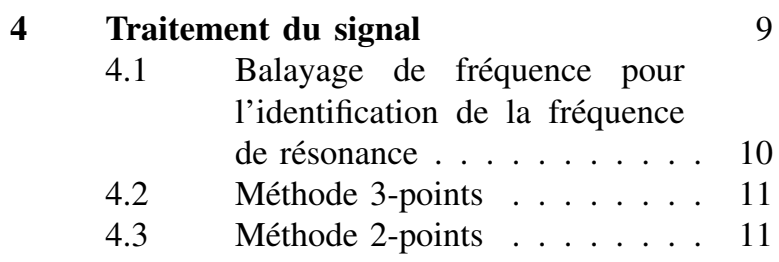

\begin{tabular}{|c|c|c|c|}
\hline 5 & Principe de sensibilité : grande & urs acces- & \\
\hline sibles & & & 12 \\
\hline & intrinsèque & vs ex- & \\
\hline & trinsèque & & 12 \\
\hline & Conception du capteur & . . . . . & 13 \\
\hline & Conception du capteur & : choix & \\
\hline & du substat $\ldots \ldots$ & $\ldots$ & 15 \\
\hline 6 & Exemples d'application & & 17 \\
\hline & Conclusion & & 20 \\
\hline
\end{tabular}

\section{Références}

\section{INTRODUCTION}

Les dispositifs passifs interrogeables sans fils répondent au besoin qu'aucune autre technologie ne peut appréhender : fournir un signal représentatif d'une grandeur physique au moyen d'un transducteur passif (sans source d'énergie locale) dont les caractéristiques sont sondées à travers une liaison radiofréquence. Ces capteurs sont déployés en environnement hostile dans lesquels une source d'énergie ne saurait fonctionner ou dans un environnement où la longévité du capteur 2 ne pourrait être garantie par une source d'énergie locale. Le principe qui préside à la conception de capteurs passifs interrogeables sans fil consiste à considérer ceux-ci comme une cible coopérative d'un système RADAR à courte portée : le RADAR se comporte comme une source de signal conçue pour sonder la réponse de la cible, dont la section RA-

5 DAR ("réponse") est affectée par son environnement 
et en particulier la grandeur physique à mesurer. Le signal rétropropagé par la cible contient l'information qui nous intéresse - la grandeur mesurée - qui est extraite après réception par le RADAR par traitement du signal. Nous nous efforçons par ailleurs de suivre deux principes qui, sans être intrinsèquement liés aux capteurs passifs interrogeables sans fils, garantissent la robustesse de la mesure : nous nous focaliserons sur les informations liées à la phase ou à la fréquence des signaux plutôt qu'à leur amplitude par soucis de robustesse et d'immunité de la réponse à l'environnement et par ailleurs nous rechercherons des mécanismes d'interaction de la cible avec le champ électromagnétique rayonné par le RADAR qui soient linéaires afin de garantir une réponse du capteur quelle que soit la puissance incidente, la portée étant alors limitée par le plancher de bruit de l'étage de réception qui détermine la plus petite puissance détectable.

\section{PRINCIPE DE MESURE}

La chaîne de mesure décrite ci-aprés détermine le plan de cet article : une source radiofréquence se doit d'émettre un signal électromagnétique qui corresponde au mieux à la réponse impulsionnelle de la cible coopérative formée du capteur. Une antenne liée au capteur couple ce champ incident avec le capteur en respectant les conditions d'adaptation d'impédance dans un environnement variable. Le capteur couple certaines composantes spectrales du signal incident dans sa bande passante et soit rétro-propage certaines composantes spectrales après avoir emmagasiné l'énergie (résonateur), soit retarde l'onde incidente (Fig. 1).
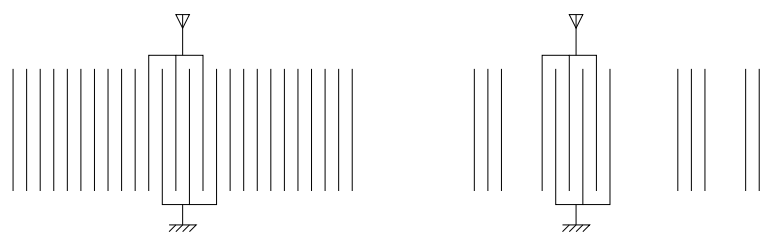

FIGURE 1. Architecture des deux géométries les plus courantes de capteurs à ondes élastiques : à droite la ligne à retard dans laquelle l'onde effectue des allers-retours entre les électrodes interdigitées et les miroirs placés sur le chemin acoustique ; à gauche le résonateur où l'onde est confinée au sein du transducteur interdigité entre les miroirs de Bragg latéraux. Noter que l'onde est émise symétriquement de part et d'autre des transducteurs interdigités dans les deux architectures, d'où la présence de mirroirs de chaque côté de ces électrodes. La réponse spectrale et temporelle de tels dispositifs est présentée en Fig. 5

Dans les deux cas, la sensibilité des caractéristiques de l'onde de surface aux conditions environnementales, en particulier sa vitesse de phase, modulent la réponse du capteur. Couplée à l'antenne par réciprocité et réversibilité du principe de transduction, celle-ci donne lieu à un signal diffusé à travers le milieu qui sépare le capteur du RADAR, pour finalement atteindre l'étage de réception. Diverses stratégies de traitement du signal, en particulier de compression d'impulsion, ont été explorées pour améliorer le rapport signal à bruit et donc abaisser la limite de détection.

\section{Aspect historique}

L'utilisation de cibles coopératives pour compléter les informations de section RADAR d'une cible illuminée par un champ électromagnétique date de la seconde guerre mondiale, avec diverses signatures introduites par les cibles lors des observations par les opérateurs de RADAR. Ce concept a été formalisé dès 1948 [1] avec la description d'une cible susceptible d'introduire une signature représentative d'un code (vitesse de rotation de réflecteurs pour moduler le signal rétrodiffusé) ou d'une grandeur physique (modulation de l'impédance de la cible). Cette publication fait antériorité à l'utilisation à des fins d'espionnage par Léon Theremin [2] d'une membrane illuminée par un RADAR pour moduler un signal incident par la voix de l'ambassadeur des État-Unis d'Amérique dans sa résidence de Moscou. Plus proche de nous, la signature RADAR des avions furtifs est amplifiée, lors de leur utilisation en dehors d'une zone de conflit, par des cibles coopératives emmagasinant puis restituant efficacement l'onde électromagnétique incidente (sphère de Lüneburg [3]), tandis que divers développements de cibles coopératives se fondant sur des couplages électromagnétiques (chipless RFID) sont explorés. Les services de renseignement sont évidemment friands de ces dispositifs passifs, n'émettant aucune signature en l'absence de sollicitation extérieure pour les interroger [4] : tous les principaux services de renseignement ont ainsi développé leur microphone passif interrogeable par excitation RADAR d'un résonateur dont la section RADAR varie avec l'intensité du champ de pression acoustique impactant la membrane servant d'électrode à un condensateur chargeant l'antenne.

On notera dès à présent que le choix d'une interaction du capteur avec le champ électromagnétique incident distingue cette approche des identifiants radiofréquences (RFID) ou récupération d'énergie (energy harvesting) qui font appel à un élément non-linéaire de redressement du courant alternatif et présentent donc un seuil minimum de démarrage : il ne s'agit donc pas de télé-alimentation mais de cible coopérative. Par ailleurs, nous effectuons une mesure analogique de laquelle est extraite la grandeur physique d'intérêt, contrairement aux capteurs numériques 
radiofréquences $(\mathrm{RF})$ qui se contentent de transmettre leur mesure sur une fréquence porteuse. Dans notre cas, le rapport signal à bruit de la mesure détermine directement l'écart type de la grandeur physique ainsi déduite. Par conséquent, la résolution de la mesure n'est pas une information figée mais dépend de la configuration de la chaîne de mesure complète. Ainsi, une approche système est nécessaire pour appréhender l'ensemble de la chaîne de mesure : se focaliser sur un seul élément ne saurait tirer le meilleur parti de l'ensemble des éléments impliqués dans l'acquisition de la caractéristique de la cible et son exploitation pour la mesure de la grandeur recherchée.

\section{ARChiteCture DE LA CIBLE COOPÉRATIVE}

\subsection{Cible coopérative : le transducteur à ondes} élastiques de surface

Les stratégies permettant de différencier la réponse du capteur de l'environnement électromagnétique conditionnent fondamentalement la conception d'un composant RF passif utilisé comme cible coopérative (Fig. 2). Les structures réfléchissantes autour du RADAR auront en général une section efficace bien plus grande que celle du capteur : il nous faut donc jouer sur le paramètre temps pour décorréler la réponse du capteur de celle de l'environnement. L'approche la plus naïve tient à retarder le signal au-delà de l'écho le plus lointain perceptible par le RADAR compte tenu du bruit de son récepteur et de la puissance émise : cette approche a été très tôt mise en œuvre avec succès, notamment par C.T. Allen [5] en utilisant une ligne à retard formée à partir d'un câble coaxial. Toutefois, retarder une onde électromagnétique RF d'une microseconde, soit le temps de vol de celle-ci au travers de $85 \mathrm{~m}$ de glace de permittivité relative de 3 (donc de célérité $300 / \sqrt{3} \simeq 200 \mathrm{~m} / \mu$ s) ou de $16 \mathrm{~m}$ d'eau de permittivité relative de 80 (donc de célérité $300 / \sqrt{80} \simeq 33 \mathrm{~m} / \mu \mathrm{s}$ ), nécessite un câble coaxial de longueur comparable aux valeurs précédentes (une centaine de mètres).

La solution n'est ni compacte, ni susceptible de fournir un capteur efficace. La conversion de l'onde électromagnétique en onde élastique - au cœur de la conception des composants de traitement du signal analogiques radiofréquences - par un substrat piézoélectrique permet d'exploiter des ondes élastiques de vitesse de phase $10^{5}$ fois plus faible que celle d'une onde électromagnétique. À fréquence fixe, la longueur d'onde est réduite d'autant et l'effet s'applique également à la longueur de la ligne à retard. Les $100 \mathrm{~m}$ de câble se trouvent réduits à $1 \mathrm{~mm}$ selon ce pricipe. Cette approche a été identifée il y a plus de 30 ans par les pionniers du domaine [6],

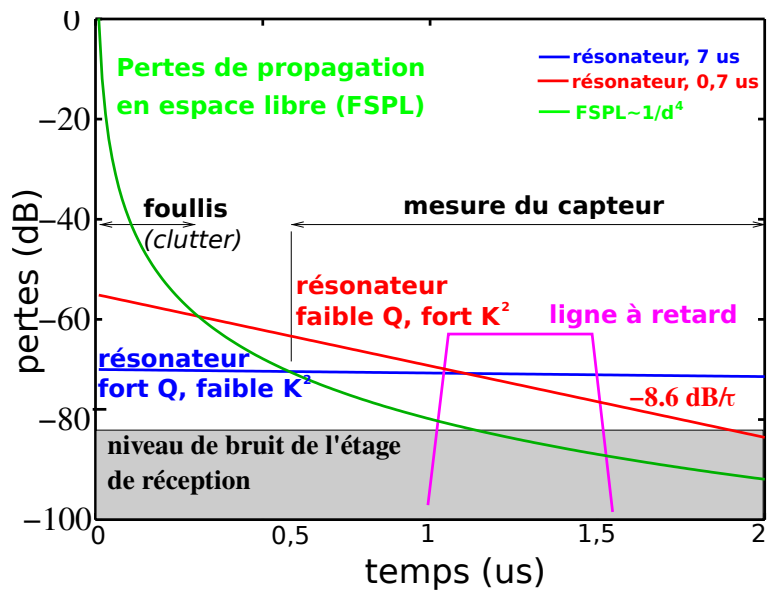

FIGURE 2. Principe de conception de la cible coopérative : le signal renvoyé par le capteur doit être suffisamment retardé pour être séparé du bruit induit par les réflexions sur les obstacles environnant le capteur mais sans être excessivement atténué pour ne pas détériorer la portée de la mesure. Cet objectif est atteint par deux architectures de transducteurs : lignes à retard ou résonateurs se déchargeant avec une constante de temps de $20 \log _{10}(e)=8,7 \mathrm{~dB} / \tau$

approfondie dans les années 1990 [7], [8], [9], [10], [11] et donne lieu à des développements commerciaux qui se poursuivent jusqu'à ce jour [12].

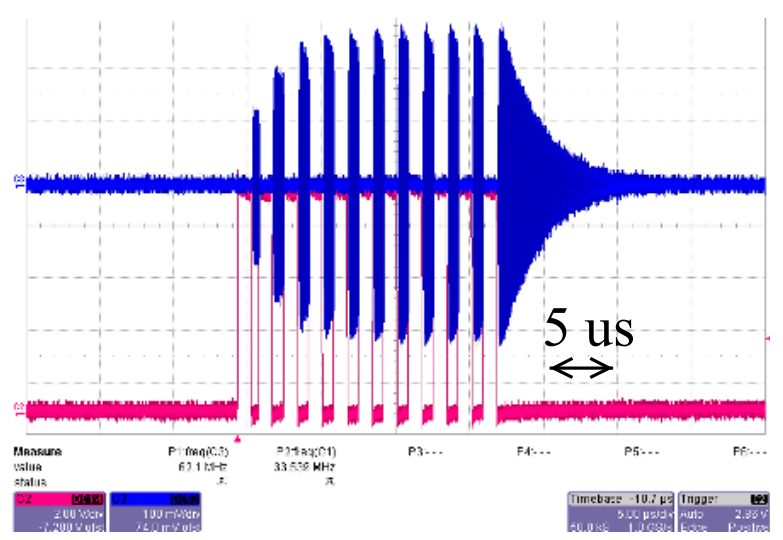

FIGURE 3. Charge et décharge d'un résonateur excité à sa fréquence de résonance. La tension aux bornes du résonateur lors de la phase de charge est découpée pour observer son évolution temporelle : dans la pratique, la porteuse est émise en continu lors de la charge pendant une durée de 5 à 10 constantes de temps du résonateur, puis coupée lors de la phase d'écoute pour observer la décroissance exponentielle du signal.

La seconde approche [13], [14], toute aussi efficace mais moins intuitive, consiste à emmagasiner l'onde élastique dans une structure résonante, en tirant parti avantageusement de substrats piézoélectriques monocristallins réputés à pertes minimale. L'énergie emmagasinée par le composant à ondes élastiques est restituée petit à petit lors de la décharge : un résonateur 
est ainsi formé d'une cavité confinant l'onde élastique entre deux miroirs - interfaces planes ou miroirs de Bragg formés d'électrodes de période moitié de la longueur d'onde - caractérisé par un facteur de qualité $Q$ définissant la fraction de l'énergie emmagasinée à l'énergie perdue par période. Dans un système simple (de type RLC série), ce comportement se traduit par une constante de temps $\tau$ de décharge du résonateur de $Q / \pi$ périodes. Cette constante de temps est idéalement supérieure aux phénomènes liés à la réflexion des ondes électromagnétiques sur les objets environnants dont la cohérence reste généralement inférieure à la microseconde. Avec un coefficient de qualité supérieur ou ègal à 10000 à $434 \mathrm{MHz}$ (cas des résonateurs à ondes de Rayleigh sur quartz), nous atteignons des constantes de temps de l'ordre de $7 \mu$ s, respectant l'objectif annoncé. La charge et décharge d'un résonateur sont illustrées sur la Fig. 3

Le choix du substrat piézoélectrique et de l'orientation cristalline doivent donc tenir compte d'une multitude de paramètres complexes à optimiser : fréquence de travail, sensibilité de la vitesse de phase de l'onde élastique à chaque grandeur considérée ou interférante, nature de l'onde (polarisation, guidage) en fonction de l'environnement de travail et des contraintes technologiques, couplage électromécanique de l'onde, facteur de qualité, impédance électrique pour les plus importants. Par exemple, il a été démontré que couplage et facteur de qualité sont deux figures de mérite antagonistes [15], [16]. Les choix de conception du transducteur modifient les caractéristiques opérationnelles du système dans son ensemble : alors qu'un résonateur présente une réponse typique de décroissance exponentielle de la puissance renvoyée avec une constante suffisamment longue pour que la réponse n'occupe qu'une bande spectrale étroite (réponse impulsionnelle théroriquement infinie), une ligne à retard génère nécessairement un signal de réponse impulsionnelle théoriquement finie couvrant une bande spectrale large incompatible avec certaines normes d'émission radiofréquences imposant un gabarit donné (par exemple la bande Industrielle, Scientifique et Médicale - ISM - autour de 433,92 MHz dont l'étendue spectrale vaut 1,7 MHz). L'impact de ce choix se retrouve sur le temps de réponse du capteur : alors qu'une charge et décharge de résonateur pendant 10 constantes de temps nécessite au moins $140 \mu \mathrm{s}$, considérée comme la durée minimale pour sonder la réponse d'un tel composant, une ligne à retard peut achever de renvoyer le signal utile $2,5 \mu$ s après avoir été sollicitée par l'impulsion incidente, offrant de ce fait un taux de rafraîchissement de la mesure considérablement plus important que celui des résonateurs. La conséquence ne concerne pas que la bande passante de mesure mais aussi la capacité de rejet des mesures erronées, voir de moyenne glissante destinée à améliorer le rapport signal à bruit de la mesure. La qualité de la mesure à portée fixe ou la portée à écart type de la mesure imposé dépendent donc des choix de conception abordés ici.

Toutes les approches classiques de partage de canal de communication ont été exploitées pour la mesure de cibles coopératives utilisées comme capteur : division des accès multiples (DMA) à répartition dans le temps (TDMA) pour les lignes à retard, division en fréquence (FDMA) pour les résonateurs, division par code (CDMA) avec encodage sur la phase de lignes à retard d'un code caractéristiques de chaque capteur [17], [18], [19]. Le multiplexage spatial (SDMA) a été envisagé [20], [21] mais n'est pas utilisé dans les applications commercialement disponibles à ce jour, compte tenu de l'encombrement excessif d'une antenne à ouverture synthétique (SAR pour Synthetic Aperture RADAR) aux relativement basses fréquences utilisées pour les capteurs à ondes élastiques, qui rendraient les dimensions de l'électronique de mesure incompatibles avec beaucoup d'applications industrielles. Par ailleurs, l'application d'une approche de SAR est d'autant plus favorable que le champ lointain est atteint, un cas rarement accessible compte tenu de la portée réduite de mesure des capteurs à ondes élastiques (quelques mètres).

L'utilisation de transducteurs modulés linéairement en période fait également partie des approches couramment utilisées pour améliorer le rapport signal sur bruit des signaux renvoyés par les capteurs. Interrogés à l'aide d'une excitation modulée linéairement en fréquence selon la même séquence que celle appliquée au transducteur, le composant fonctionne alors comme un corrélateur et renvoie un signal compressé dans le domaine temporel. Cette approche permet d'améliorer le contraste de la réponse en donnant lieu à des niveaux de pertes de l'ordre de $16 \mathrm{~dB}$ contre 25 à 30 au mieux pour des systèmes sans modulation. Différentes options sont envisageables dans ce même esprit, tel que l'utilisation de code de phase [22] pour donner une spécificité spectrale à la réponse impulsionnelle du capteur. Dans l'idée d'ajuster la fonction de transfert du dispositif aux variations de la grandeur physique à mesurer, une distribution hyperbolique plutôt que linéaire des électrodes au sein du transducteur a été proposée [23]. Contrairement au cas du corrélateur linéaire présenté plus haut, celui-ci permet la fonction de corrélation quelles que soient les variations de la 
grandeur physique d'intérêt. Accessible jusqu'à $2 \mathrm{GHz}$ pour les composants à ondes de surface, cette approche se heurte aux limites technologiques en vigueur dans le domaine pour des fréquences dépassant les $2 \mathrm{GHz}$ (résolution des systèmes de lithographie de l'industrie des SAW limitée à $350 \mathrm{~nm}$ typiquement).

Plus originales, des architectures de capteurs répondant aux exigences d'anti-collision des réponses de plusieurs dispositifs en vue simultanée du système de mesure ont été développées en exploitant des séquences orthogonales de fréquence (OFDM) [24], [19].

\subsection{Bilan de liaison}

Un bilan de liaison RADAR classique fournit une estimation de la puissance radiofréquence reçue connaissant la puissance radiofréquence émise, la distance entre l'électronique de mesure (configuration monostatique) et la cible, la section RADAR de cette dernière et le gain des antennes. Cette approche est valable pour des cibles de section efficace caractérisée et dont la dépendance spectrale ne varie pas significativement. Les cibles coopératives à ondes élastiques de surface présentent une bande passante faible face à celle des impulsions RADAR, et l'adaptation du signal d'interrogation à la fonction de transfert du capteur est un point clé du bilan de liaison. Par ailleurs, la désadaptation d'impédance entre l'antenne soumise à un environnement variable et le capteur dont les paramètres équivalents évoluent avec les conditions environnementales, influe elle aussi sur le bilan de liaison. Finalement, contrairement à la propagation d'une onde électromagnétique dans l'air mais en cohérence avec la modélisation des RADAR de sol, le capteur peut être enfoui dans un matériau (polymère, béton, sol) qui présente une conductivité non nulle et donc des pertes de propagation dont il faut tenir compte dans le bilan de liaison.

Le point clé déterminant la porée de la mesure dans une liaison RADAR est l'isolation : la puissance reçue décroît comme la puissance quatrième de la distance entre l'antenne du RADAR monostatique et la cible en supposant cette dernière ponctuelle - pour atteindre une dynamique système de $60 \mathrm{~dB}$ de $2 \mathrm{~m}$ à $434 \mathrm{MHz}$ ou $92 \mathrm{~dB}$ sur une même distance à $2,4 \mathrm{GHz}$ à la même distance. Tout signal fuyant de l'émetteur vers le récepteur avec une puissance relative plus élevée cachera le signal renvoyé par le capteur.

Dans le bilan de liaison RF, la section RADAR $\sigma$ détermine la surface équivalente d'un réflecteur illuminé par le signal incident et donc la puissance reçue une fois normalisée par la longueur d'onde $\lambda$. Lors de l'interrogation d'un dispositif à ondes élastiques, le terme $\sigma / \lambda^{2}$ est remplacé par les pertes d'insertion $I L$ dans le domaine temporel (après transformée de Fourier inverse de la réponse du dispositif dans le domaine spectral tel qu'obtenue avec un analyseur de réseau). Pour une ligne à retard sub-GHz, les pertes d'insertion sont typiquement de 20 à $30 \mathrm{~dB}$. Une telle grandeur se déduit, pour une ligne à retard, par la transformée de Fourier inverse de la caractérisation spectrale, par analyseur de réseau, du coefficient de réflexion $S_{11}$ du capteur. Pour un résonateur, la puissance réfléchie est le complément, par conservation de l'énergie, de la puissance absorbée par le dispositif, soit $1-\left|S_{11}\right|$.

\section{3 ÉleCtronique de MESURE}

L'architecture de la cible coopérative étant définie et ses caractéristiques spectrales et temporelles déterminées, il s'agit maintenant de savoir comment sonder la réponse de ce transducteur par une liaison radiofréquence (Fig. 4). Le RADAR à courte portée répond à l'exigence de caractériser la réponse de la cible coopérative par une liaison sans fil sans qu'une source d'énergie ne soit nécessaire à proximité de l'élément sensible. Le pendant des deux grandes architectures de capteurs - résonateurs ou lignes à retard - en terme d'électronique de mesure sont les déclinaisons dans les domaines temporel ou spectral de la mesure. Nous avons mentionné que ces deux stratégies de mesures sont duales au travers de la transformée de Fourier et seuls la technologie et le respect des normes favorisent l'une ou l'autre approche.

Toutes les approches de RADAR à courte portée sont exploitables pour interroger les capteurs [25] : impulsionnel, onde continue balayée ou onde continue émise par paliers, à bruit par la génération d'un code pseudo-aléatoire pour étaler le spectre ou exploitant des sources non-coopératives dans une approche de RADAR passif [26], [27], [28]. Alors que toutes ces méthodes sont théoriquement équivalentes par transformée de Fourier, les conditions de déploiement opérationnel pourront pousser à sélectionner une approche ou l'autre.

L'approche impulsionnelle est la plus intuitive et celle qui fournit la meilleure isolation entre émetteur et récepteur et donc a priori la meilleure portée si le couplage émetteur-récepteur limite la portée (et non le bruit sur l'étage de réception). Dans la pratique, la stratégie temporelle qui consiste à émettre une impulsion brève - donc de large encombrement spectral - et à échantillonner les échos renvoyés par les cibles illuminées avec une fréquence d'échantillonnage plus importante que la largeur spectrale de l'impulsion émise, n'est utilisée dans aucune solution commerciale 


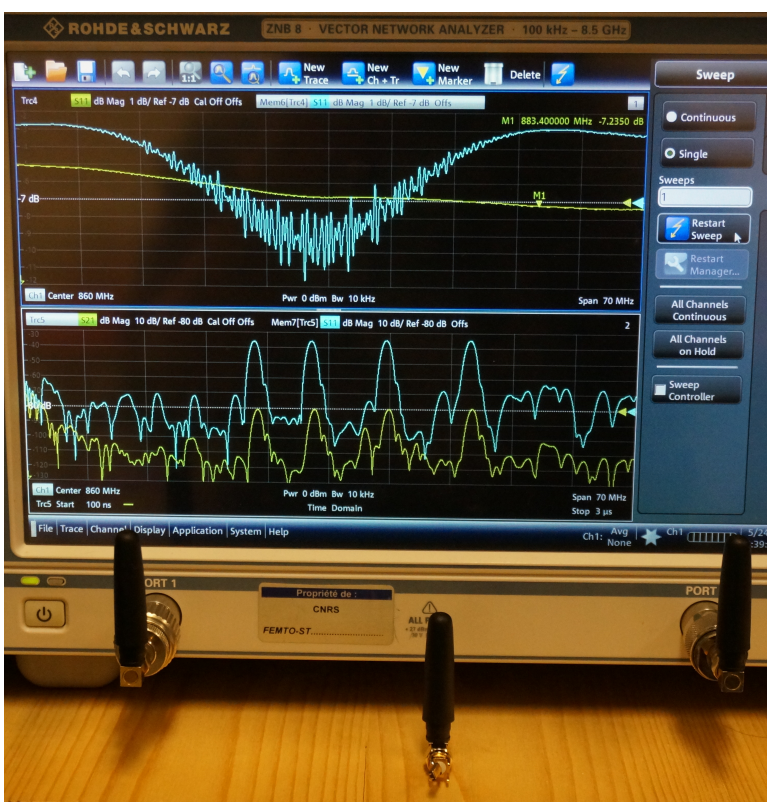

FIGURE 4. Alors que les systèmes électroniques de mesure dédiés s'apparentent à des RADAR courte portée visant à séparer le signal émis du signal rétrodiffusé par la cible, un montage aussi simple qu'un analyseur de réseau suffit pour une première approche expérimentale. La mesure en transmission $\left(\mathrm{S}_{21}\right)$ permet un gain d'isolation qui se traduit par un gain en portée par rapport à une mesure en réflexion. En bleu la caractérisation en mode conduit en réflexion d'une ligne à retard fonctionnant autour de $870 \mathrm{MHz}$, et en vert la mesure sans fil en transmission. En haut la réponse spectrale, en bas la mesure temporelle obtenue par transformée de Fourier inverse de la réponse spectrale. Noter que la réponse temporelle ne commence qu'à $100 \mathrm{~ns}$ pour éliminer le couplage direct nettement plus puissant que le signal rétrodiffusé par la cible coopérative située entre les deux ports de l'analyseur de réseau.

de lecteur de dispositifs à ondes élastiques. Cette approche [29] est cependant intéressante car elle permet d'atteindre le taux maximal de rafraîchissement de la mesure, uniquement limité par la durée d'écoute déterminé par l'écho le plus long renvoyé par le capteur. Ainsi, pour des échos situés entre 1 et $2 \mu \mathrm{s}$, une mesure à plus de 500 kéchantillons/s est accessible. Une telle performance ne présente pas de réel intérêt pour analyser une grandeur variant lentement (quelques $\mathrm{Hz}$ à quelques centaines de $\mathrm{Hz}$ ) telle que la température, si ce n'est dans un environnement bruité pour rejeter les trames polluées par des émissions parasites ou pour permettre de moyenner sur un grand nombre de mesures pour réduire la variance sur la grandeur observée mais présente un réel intérêt pour des mesures vibratoires.

L'inconvénient de ces méthodes rapides de mesure est de nécessiter une grande bande passante qui se traduit par une consommation électrique importante généralement peu compatible avec les applica- tions embarquées. Une alternative bien connue dans la mise en œuvre des RADARs à pénétration de sol (Ground Penetrating RADAR - GPR) - RADAR impulsionnel classiquement exploités en géophysique et parfaitement adaptés pour sonder la réponse de capteurs passifs enterrés [30], [31], [32], [33], est le récepteur stroboscopique. Dans cette approche, une impulsion radiofréquence est émise, et un unique échantillon mémorisé par un échantillonneur-bloqueur rapide pour permettre une conversion lente grâce à un convertisseur analogique-numérique de bande passante et consommation électrique réduites et de résolution supérieure à celle accessible pour les convertisseurs radiofréquences. Cette mesure est répétée pour autant de points de mesure requis avec un échantillonnagebloquage décalé dans le temps par un pas inverse de la fréquence équivalente de conversion qui peut ainsi atteindre plusieurs dizaines de gigahertz malgré un convertisseur nécessitant plusieurs microsecondes pour fournir une donnée. Dans ce cas, le gain en bande passante sur la mesure du RADAR impulsionnel est perdu par l'approche stroboscopique exploitée sur l'étage de réception.

Contrairement aux applications RADAR qui sondent une cible de bande passante très large et cherchent à en identifier la position par temps de vol de l'impulsion émise, la mesure d'un capteur nécessite de générer une impulsion dont l'encombrement spectral est commensurable avec celui de la cible. Or un capteur présente une bande de fonctionnement (Fig. 5) de l'ordre du coefficient de couplage électromécanique $k_{s}^{2}$ de l'onde, soit de quelques pourcents à une fraction de pourcent selon les substrats piézoélectriques. Ainsi, seule une fraction de l'impulsion ultralarge bande (définie comme un encombrement spectral de $500 \mathrm{MHz}$ ou $20 \%$ de la fréquence centrale de fonctionnement selon la valeur la plus petite [34], [35], [36]) formée d'une impulsion unique est couplée au capteur, puisque toute l'énergie hors bande est immédiatement réfléchie sans être convertie en onde élastique. Ainsi, le nombre de périodes dans l'impulsion sera idéalement de l'ordre de l'inverse du couplage électromécanique, soit une vingtaine de paires d'électodes pour l'onde de Rayleigh sur la couple (YXl)/128 ${ }^{\circ}$ du niobate de lithium ou de plusieurs centaines pour cette même onde sur la coupe AT de quartz. À cette première estimation, il faut ajouter le besoin d'adapter l'impédance électrique du composant à sa charge rayonnante (antenne) qui peut modifier ces ordres de grandeur de façon sensible. Pour un accord à $50 \Omega$, 15 paires d'électrodes constituent un optimum pour 
le niobate $(\mathrm{YX} / \mathrm{l}) / 128^{\circ}$ par exemple.

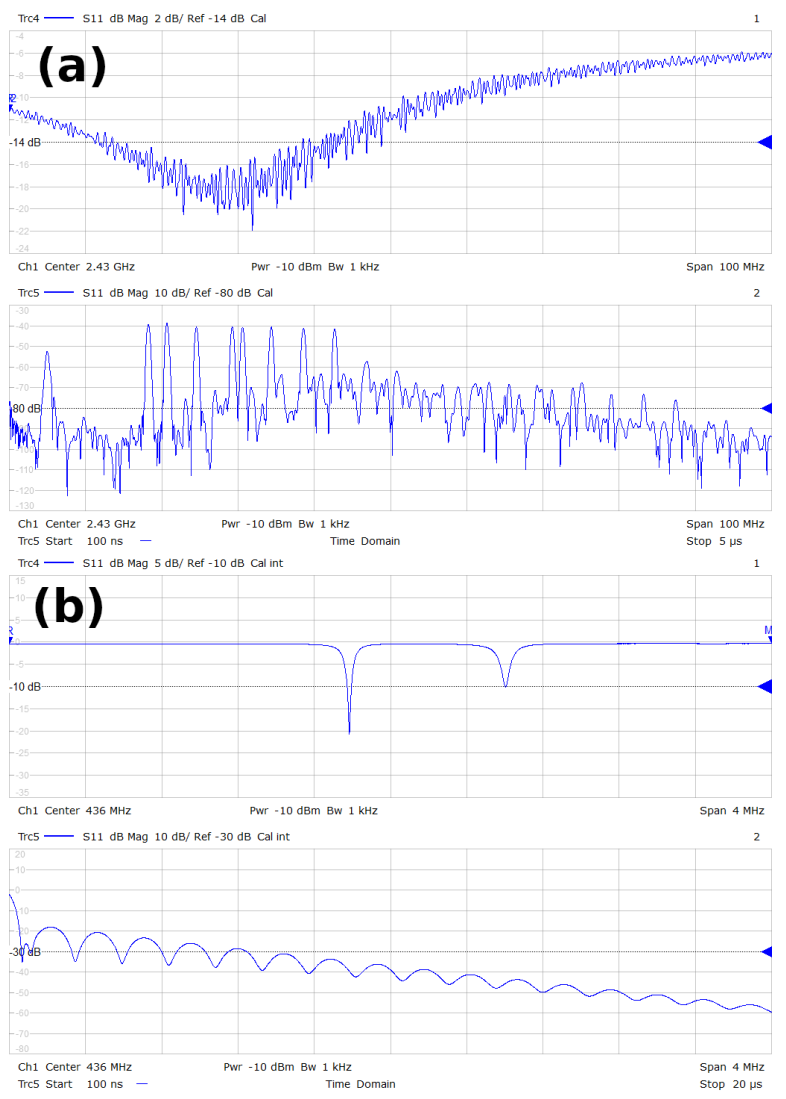

FIgURE 5. Réponses spectrale (haut) et temporelle (bas) des deux architectures de transducteurs : ligne à retard (a) et résonateur (b). Le résonateur est caractérisé sur la gamme de fréquences $4 \mathrm{MHz}$ autour de $436 \mathrm{MHz}$ avec une échelle de $5 \mathrm{~dB} /$ division pour la caractérisation spectrale (haut), et de 0,1 à $20 \mu$ s avec une échelle de $10 \mathrm{~dB}$ /division pour la caractérisation dans le domaine temporel (bas). La ligne à retard est caractérisé sur la gamme de fréquences $100 \mathrm{MHz}$ autour de $2430 \mathrm{MHz}$ avec une échelle de $2 \mathrm{~dB}$ /division pour la caractérisation spectrale (haut), et de 0,1 à $5 \mu$ s avec une échelle de $10 \mathrm{~dB} /$ division pour la caractérisation dans le domaine temporel (bas).

L'alternative aux approches larges bandes (impulsionnelles) sont les approches à bandes étroites, moins gourmandes en ressources radiofréquences sur l'étage de réception mais nécessitant des durées d'interrogation plus longues en sondant séquentiellement les diverses contributions spectrales : balayage de fréquence par pas discrets (Frequency Stepped Continuous Wave - FSCW) tel que classiquement mis en œuvre au sein des analyseurs de réseau ou balayage continu (Frequency Modulated Continuous Wave - FMCW) dans lequel le signal retardé dans le temps est mélangé avec le signal émis selon une modulation de fréquence linéaire [37]. Dans ce dernier cas, la fréquence de battement en sortie du mélangeur est proportionnelle au retard introduit par la cible, qui est donc directement obtenu par transformée de Fourier [38].
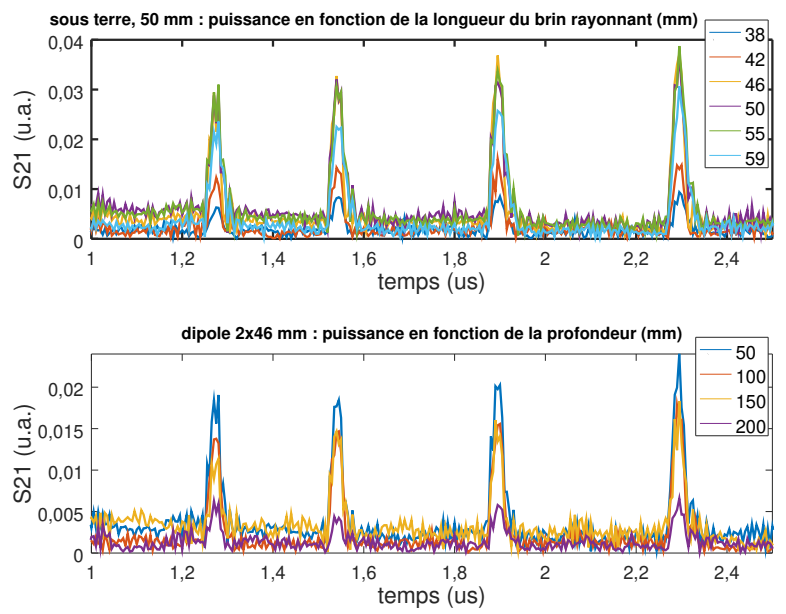

FIGURE 6. Mesure bistatique par balayage de fréquence par un analyseur de réseau configuré pour la mesure du paramètre de diffraction $S_{21}$ en transmission dans la bande spectrale de fonctionnement des transducteurs inter-digités et transformée de Fourier inverse pour l'obtention de la réponse temporelle. La ligne à retard centrée sur $856 \mathrm{MHz}$ et de $20 \mathrm{MHz}$ de bande passante correspond au capteur utilisé par le système SENTRY de Kongberg. Les légendes indiquent la demi-longueur de dipôle (haut, donc longueur de chaque brin rayonnant, en $\mathrm{mm}$ ) ou la profondeur du capteur enterré dans le sable (bas, en $\mathrm{mm}$ ). Noter que les dimensions optimales du dipôle, entre 45 et $55 \mathrm{~mm}$ pour chaque brin, correspond à la valeur théorique du quart de la longueur d'onde pour un milieu de permittivité relative entre 1,3 et 1,6 , raisonnable pour du sable sec [39].

L'approche à balayage de fréquence en onde continue (FMCW) exploite le battement entre l'onde transmise à un instant et l'onde renvoyée par le capteur avec un retard $d t$. Pour une excursion en fréquence $\Delta f$ balayée en un temps $\Delta t$, le retard $d t$ introduit donc un battement de fréquence $\Delta f \times d t / \Delta t$. En choisissant $\Delta t$ de l'ordre du millième de $d t-$ par exemple respectivement $1 \mathrm{~ms}$ pour un retard des échos de l'ordre de $1 \mu \mathrm{s}-$ la fréquence de battement passe de la bande radiofréquence $(\mathrm{MHz})$ à la bande audiofréquence $(\mathrm{kHz})$ beaucoup plus simple à acquérir et traiter : une telle réalisation peut se contenter d'une carte son d'ordinateur personnel (192 kéchantillons/s avec une résolution pouvant aller jusqu'à 24 bits pour les meilleurs modèles) pour réaliser l'étage d'acquisition après le mélangeur. Le point clé de cette implémentation est la linéarité de la fréquence émise par la source en fonction du temps : parmi les diverses solutions de sources de fréquences agiles que sont les oscillateurs contrôlés en tension (VCO), les boucles à verrouillage de phase (PLL) ou les synthétiseurs de fréquence directs (Direct Digital Synthesizer - DDS), seuls les derniers garantissent une linéarité de leur sortie lors de leur programmation par une rampe linéaire 
de fréquence. Un VCO nécessite une compensation de la non-linéarité de sa fréquence de sortie en fonction de sa tension d'entrée si une vaste plage de fréquence est abordée et une PLL nécessite un temps de stabilisation excessive.

L'approche à pas de fréquences en onde continue (FSCW) caractérise la réponse spectrale de la cible par pas discrets de fréquence, dont la transformée de Fourier inverse fournira la réponse temporelle. Si les fréquences sondées par le RADAR à courte portée sont espacées de $d f$, alors le temps total de mesure $\Delta t$ analysé est $1 / d f$ et si $N$ points sont acquis dans le domaine spectral (excursion spectrale $\Delta f=N \times$ $d f$ ), alors la résolution temporelle de la mesure sera $1 / \Delta f=\Delta t / N$. Une application numérique indique que si un temps de mesure de l'ordre de $2,5 \mu$ s est requis pour une excursion spectrale de $20 \mathrm{MHz}$ de la bande passante du capteur, alors le nombre de points à acquérir est de $2500 / 50=500$ points puisque $50 \mathrm{~ns}$ est l'inverse de $20 \mathrm{MHz}$ et $2500 \mathrm{~ns}$ est la durée totale de mesure. Dans la pratique, on s'autorisera un peu de marge de manœuvre en augmentant le nombre de points, bien que les conséquences d'un tel choix portent principalement sur le niveau de corrélation entre courbes théorique et expérimentale destiné à améliorer le rapport signal à bruit sans véritables critères quantifiés a priori.

La première méthode est celle utilisée dans la majorité, si ce n'est la totalité, des RADARs de sol pour les mesures sub-surfaces d'interfaces, dont nous illustrerons l'utilisation pour des capteurs enterrés en section 6. La seconde méthode permet une démonstration rapide (Fig. 4) et faible coût de la mesure de lignes à retards mais n'est pas exploitée commercialement à notre connaissance. La troisième méthode est déclinée en stratégie de mesure pour lignes à retard (avec transformée de Fourier inverse de la réponse spectrale) ou pour les résonateurs (observation directe de la fréquence de résonance dans le domaine spectral). Une extension de celle-ci porte le nom de time gated FSCW dans laquelle l'onde réfléchie par le capteur ne commence à être enregistrée que quelques nanosecondes après la fin de l'émission afin de minimiser le couplage direct qui saturerait l'étage de réception (Fig. 7) [40]. Cette approche est utilisée par le lecteur de résonateurs de SENSeOR ou le lecteur de lignes à retard de RSSI. Les méthodes par balayage de fréquence profitent des évolutions technologiques de synthèse de signaux radiofréquences et en particulier la synthèse numérique directe DDS qui permet de sauter "instantanément" (avec un délai uniquement déterminé par le temps de programmation du composant numérique et non un temps de stabilisation de boucle d'asservissement tel que nous le rencontrons dans une boucle à verrouillage de phase) d'une fréquence à une autre. De ce fait, des algorithmes permettant de s'adapter à la réponse du capteur sont envisageables au-delà du simple balayage de la bande de fréquence contenant la réponse du dispositif. Ces méthodes sont présentées en section 4.1.

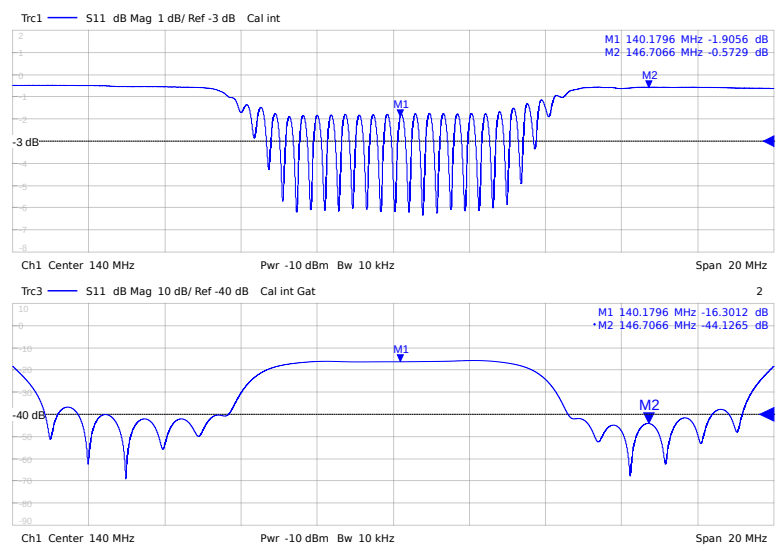

FIGURE 7. Gain en isolation par découpage temporel de la phase d'émission du signal radiofréquence d'interrogation et de la phase de réception : en haut la mesure de $S_{11}$ présente des absorptions lorsque la puissance électromagnétique est convertie en puissance acoustique par le transducteur acoustique, réduisant la puissance retournée vers l'analyseur de réseau; en bas, le découpage temporel (time gating) élimine l'onde électromagnétique couplant en quelques nanosecondes entre émission et réception, pour présenter la restitution de la puissance acoustique vers le port de mesure de l'analyseur de réseau comme un maximum de puissance dans la bande de fonctionnement du dispositif. Noter qu'en l'absence de découpage temporel (haut) l'isolation n'est que de $5 \mathrm{~dB}$, contre $30 \mathrm{~dB}$ en rejetant le signal direct en ne conservant le signal réfléchi qu'entre $100 \mathrm{~ns}$ et $5 \mu$ s (bas). Cette réponse est celle d'un filtre en transmission EPCOS B3607 centré sur $140 \mathrm{MHz}$ utilisé ici comme ligne à retard réflective.

La maîtrise de l'encombrement spectral de l'impulsion émise est un élément fondamental du bilan de liaison. Les méthodes impulsionnelles ne permettent pas un tel contrôle : une impulsion très large bande est générée et filtrée par l'antenne. Cette antenne voit ses caractéristiques et en particulier sa fréquence de résonance, varier avec l'environnement. Cet effet est particulièrement spectaculaire avec le RADAR de sol dont l'antenne est en contact intime avec des milieux de permittivité variable en fonction de la nature du sol mais est valable pour toute antenne dans un environnement variable. De ce fait, le bilan de liaison évolue avec cet environnement : un RADAR impulsionnel travaille à longueur d'onde imposée par les dimensions géométriques de l'antenne et donc à fréquence centrale variable si la célérité de l'onde électromagnétique varie. Cette condition est défavorable pour sonder un capteur dont la fréquence de fonctionnement est 
imposée par la géométrie des électrodes sur le substrat piézoélectrique et qui ne varie que de quelques centaines de ppm selon l'environnement du capteur (pour rappel, une antenne exposée à l'air ou plongée dans l'eau voit quant à elle sa fréquence centrale de fonctionnement varier d'un facteur 9 comme racine de la permittivité relative de l'eau de 80). Au contraire, une approche spectrale (FSCW, FMCW) impose la fréquence du signal émis et donc garantit une maîtrise de l'occupation du spectre, au détriment du bilan de liaison si la caractéristique de l'antenne dévie trop de la fréquence de fonctionnement nominale.

Enfin, les problèmes de certification du système de mesure et de l'encombrement du spectre radiofréquence sont appréhendés par les approches de RADAR passif dans lesquelles des sources noncoopératives de signaux électromagnétiques illuminent une cible coopérative. Par une conception judicieuse qui adapte la bande spectrale de fonctionnement du capteur au spectre émis, la réponse impulsionnelle du capteur est identifiée par intercorrélation du signal émis par la source non-coopérative avec le signal reçu du capteur. La résolution temporelle reste déterminée par l'inverse de la bande passante du signal illuminant la cible : le concept a été efficacement démontré par exemple avec WiFi [26]. Malgré le retard important des échos induits par le transducteur à onde élastique, la principale difficulté réside dans l'autocorrélation des signaux illuminant le capteur : les sources noncoopératives ne sont pas des sources de bruit mais présentent une structure aux retards des échos du capteur qui doit être éliminée pour permettre de retrouver le signal d'amplitude moindre du transducteur à onde élastique. Il s'agit de la suppression du signal interférant direct (Direct Signal Interference) bien connu dans le domaine du RADAR passif. Néanmois, un traitement adéquat du signal par corrélation, avec une durée d'intégration suffisamment longue - de quelques centaines de millisecondes - permet une mesure à plusieurs kilomètres de la source non-coopérative [28].

\section{TRAITEMENT DU SIGNAL}

La mesure du canal de communication radiofréquence, incluant les échos induits par les cibles coopératives, fournit une séquence de valeurs complexes, classiquement nommées I et $\mathrm{Q}$ pour les grandeurs en identité et en quadrature. Ces grandeurs sont classiquement converties en module $\sqrt{I^{2}+Q^{2}}$ et phase $\varphi=\arctan (Q / I)$ pour extraire une information sur les propriétés physiques du signal. Nous verrons dans les propriétés des matériaux composant les substrats piézoélectriques (section 5.3) que les variations relatives de fréquence, phase ou temps de vol, ne sont au plus que de quelques dizaines de parties par million (ppm) par rapport à la grandeur mesurée (température en $\mathrm{K}$, contrainte en $\mathrm{MPa}$ ). La mesure de ces variations minimes - permettant de respecter l'encombrement spectral des bandes radiofréquences selon les normes en vigueur - impose d'une part une stratégie minimisant les variations de l'oscillateur local servant de référence et d'autre part de développer des stratégies de traitement du signal prenant en compte les spécificiés de l'interrogation de dispositifs à ondes élastiques de comportement spectral et temporel parfaitement connus.

Le premier point, la sensibilité de l'oscillateur local, est en partie réduit par l'approche de mesure différentielle. En effet, alors que sur une mesure de fréquence de résonance, observer des variations de quelques ppm. $\mathrm{K}^{-1}$ à $434 \mathrm{MHz}$ nécessite un oscillateur local de stabilité long terme meilleure que $10^{-6}$, une mesure différentielle de deux modes se propageant sur un même substrat subissant les mêmes variations thermiques mais séparés de $1 \mathrm{MHz}$ permet de réduire la contrainte sur la stabilité de l'oscillateur local d'un facteur voisin de la fréquence de résonance moyenne des deux résonateurs - de l'ordre de $4,3 \cdot 10^{8}$ dans le cas illustré ici. De ce fait, la contribution de l'oscillateur cadençant le RADAR à courte portée d'interrogation de résonateurs devient négligeable face à d'autres sources de variations des propriétés physiques du canal de transmission que sont par exemple les impédances parasites dans un environnement variable (tirage de fréquence du couple antenne+capteur). Ceci reste vrai dans la limite d'oscillateurs locaux cadençant les électroniques de mesures de stabilité commensurable avec la dérive des capteurs : des sources trop dégradées, telles que présentes par exemples sur certains GPR [41], domineront la réponse du système de mesure et celle du capteur et rendront toute analyse de la grandeur physique impossible.

En supposant l'oscillateur local cadençant le RADAR de mesure suffisamment stable, l'analyse du module de l'enveloppe des échos renvoyés par une ligne à retard est trop grossière pour une mesure précise de temps de vol permettant de remonter à une grandeur physique. À titre d'exemple, deux échos séparés de $\tau=1 \mu$ s sur un substrat présentant une sensibilité en température de $S=60 \mathrm{ppm} . \mathrm{K}^{-1}$ induiront une variation de temps de vol de $\Delta \tau=60$ ps pour une variation de température de $1 \mathrm{~K}$. Identifier un tel retard nécessiterait une bande passante de mesure de l'ordre de $17 \mathrm{GHz}$, bien au-delà des quelques $80 \mathrm{MHz}$ disponibles dans la bande ISM autour de la porteuse $f=2,4 \mathrm{GHz}$. Bien plus précise que la 
mesure du module, l'analyse de la phase [42] permet de gagner un facteur 1000 sur la résolution de la mesure, en considérant que la rotation de phase induite par la propagation de l'onde élastique est $\varphi=2 \pi f \tau$. Ainsi, $S=\Delta \varphi / \varphi=\Delta \tau / \tau$ permet de déterminer la variation de phase $\Delta \varphi$ nécessaire pour observer une variation unitaire de la grandeur physique analysée : dans l'application numérique précédente, une variation de température de $1 \mathrm{~K}$ se traduit par une variation de phase de $\Delta \varphi=S \varphi=0,9 \mathrm{rad}$ ou $50^{\circ}$. Une telle mesure de phase est aisément accessible avec un détecteur I/Q transposant en bande de base le signal radiofréquence acquis.

Pour un résonateur, la variation des propriétés du résonateur et en premier lieu de la vitesse de phase du mode - combinaison de la dilatation géométrique du substrat et de l'évolution des constante élastiques du matériau qui le compose - entraîne une variation de fréquence de résonance, à longueur d'onde imposée par la géométrie des électrodes interdigitées et des mirroirs de Bragg. Deux contraintes sur la variation de fréquence en fonction de la grandeur physique à mesurer sont imposées par l'électronique de mesure et les normes d'émission radiofréquences associées. D'une part, cette variation doit être suffisamment importante pour dominer toute variation de l'oscillateur local à l'électronique de mesure qui fournit le signal de référence servant à sonder la réponse du capteur. La solution pour amplifier l'impact d'une grandeur physique sur la mesure de fréquence de résonance consiste à sélectionner une approche différentielle, dans laquelle deux résonateurs présentant des sensibilités différentes sont connectés électriquement au niveau de l'élément sensible et la différence de fréquence entre ces deux dispositifs est mesurée. Ainsi, avec deux résonances situées dans la bande industrielle, scientifique et médicale (ISM) de 433,05 à 434,79 MHz, une différence de fréquence entre ces deux résonateurs de l'ordre de $1 \mathrm{MHz}$ permet de réduire d'un facteur 434 le besoin de stabilité sur l'oscillateur de référence cadençant la source radiofréquence de l'interrogateur qui sonde la réponse du capteur. D'autre part, l'étroitesse de la bande ISM impose une seconde contrainte sur l'amplitude de variation des fréquences absolues des deux résonateurs qui doivent rester localisées dans la bande autorisée. En effet en reprenant le cas du niobate de lithium $(\mathrm{YXl}) / 128^{\circ}$ et ses $60 \mathrm{ppm} . \mathrm{K}^{-1}$ de sensibilité thermique, un résonateur centré sur $434 \mathrm{MHz}$ varie de $26 \mathrm{kHz} . \mathrm{K}^{-1}$ ou 2,6 MHz sur une plage de mesure de $100 \mathrm{~K}$, supérieure à la largeur de bande allouée. Le choix du quartz avec ses orientations cristallines compensées en température pour faire office de référence et des variations de température suffisamment faibles (quelques ppm. $\mathrm{K}^{-1}$ ) pour rester dans la bande ISM, même sur une vaste plage de températures, est ainsi naturel. Le résonateur commercialisé par SENSeOR présente ainsi une sensibilité diffŕentielle de l'ordre de $2,5 \mathrm{kHz} . \mathrm{K}^{-1}$ sur une plage de $150 \mathrm{~K}$ (Fig. 8).
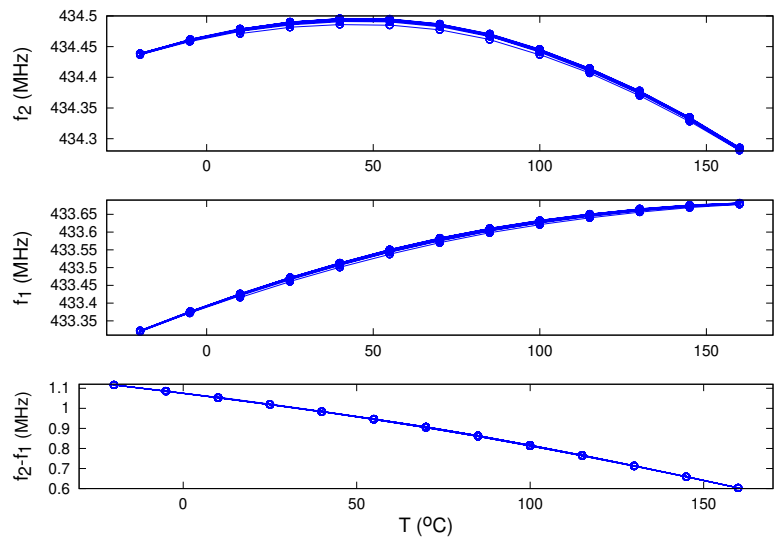

FIGURE 8. Exemple d'étalonnage d'un capteur différentiel formé de deux résonateurs sur quartz. Les deux courbes du haut (référence) et du milieu (mesure) présentent la dépendance en température de la fréquence de résonance de chaque mode et la courbe du bas présente la dépendance en température de la différence des fréquences telle qu'exploitée pour une mesure par une liaison radiofréquence.

\subsection{Balayage de fréquence pour l'identification de la fréquence de résonance}

L'approche la plus simple pour identifier la fréquence de résonance d'un résonateur consiste à le solliciter par une onde radiofréquence centrée sur la fréquence analysée. Une fois le résonateur chargé (Fig 3), l'émission est coupée et l'antenne du RADAR monostatique servant à sonder la réponse du capteur est commutée vers l'étage de réception. Dans sa mise en œuvre la plus simple cet étage de réception peut être un détecteur de puissance ou, dans une version plus évoluée un détecteur amplitude-phase référencé sur la source qui a émis l'impulsion sonde. Dans les deux cas, la connaissance de la fréquence du signal émis et la linéarité du principe d'excitation du capteur garantissent un signal renvoyé par le transducteur à la même fréquence que le signal émis : aucune analyse spectrale n'est nécessaire sur l'étage de réception si l'encombrement spectral de l'impulsion sonde est inférieur à la largeur de la résonance du capteur (impulsion longue). Cette approche, sélectionnée par des sociétés comme SENSeOR, Miele, RSSI ou encore Environetics se distingue de l'approche proposée par Transense qui au contraire émet une impulsion brève pour couvrir un encombrement spectral supérieur à la 
largeur de la résonance et analyse le signal restitué par transformée de Fourier [43], nécessitant une mémoire et une puissance de calcul nettement supérieure à l'approche par balayage de fréquence.

Une fois la fonction de transfert acquise en ayant balayé toutes les fréquences définissant la bande occupée par le capteur, un ajustement parabolique de la résonance permet d'améliorer la résolution de mesure de la fréquence de résonance. Cet ajustement parabolique souffre d'un biais introduit par l'asymétrie de la réponse spectrale d'un résonateur à ondes élastiques du fait de l'anti-résonance (section 5.2), tandis que le bénéfice de l'ajustement parabolique est d'autant meilleur que les points de mesure sont largement distribués de part et d'autre de la résonance. Nous avons démontré [44] que le pas de fréquence optimal de balayage vaut le tiers de la largeur à mi-hauteur de la résonance du capteur. À titre d'application numérique, un résonateur de facteur de qualité de l'ordre de $Q=10000$ à $f=434 \mathrm{MHz}$ présente une largeur à mi-hauteur voisine de $43 \mathrm{kHz}$ et balayer les $1,74 \mathrm{MHz}$ de la bande ISM par pas de $d f=15 \mathrm{kHz}$ nécessite 120 points de mesure. Si chaque charge et décharge de résonateur durent 5 constantes de temps $Q /(\pi f)$, alors la durée d'un balayage est $8,8 \mathrm{~ms}$, soit un taux de rafrâichissement de l'information de la grandeur mesurée de $114 \mathrm{~Hz}$ (Fig. 9p. Un tel résultat est généralement suffisant pour une mesure de température mais insuffisant pour une mesure de contrainte ou de vibration.

\subsection{Méthode 3-points}

La souplesse de la synthèse numérique par DDS permet de s'affranchir de la majorité des mesures de la réponse du capteur aux fréquences que nous savons loin de la dernière résonance mesurée, pour ne nous focaliser que sur les quelques fréquences de part et d'autre de la dernière mesure. Afin de permettre l'ajustement parabolique, 3 points sont considérés : la dernière fréquence de résonance connue suite à la mesure précédente, un point à une fréquence plus élevée d'un pas de fréquence $d f$ et un point à une fréquence en-deçà de la dernière résonance connue d'un pas de fréquence $d f$. De ce fait, les 120 points de mesure sont remplacés par seulement 3 acquisitions, ou par 6 mesures pour une approche différentielle. Dans les mêmes conditions que précédemment, le gain en vitesse de rafraîchissement de la mesure est de 20 : nous atteignons ainsi une fréquence de rafraîchissement de l'information au delà des $2 \mathrm{kHz}$. Dans ces conditions, le protocole de communication numérique entre l'électronique de mesure et un ordinateur chargé d'acquérir les informations peut devenir

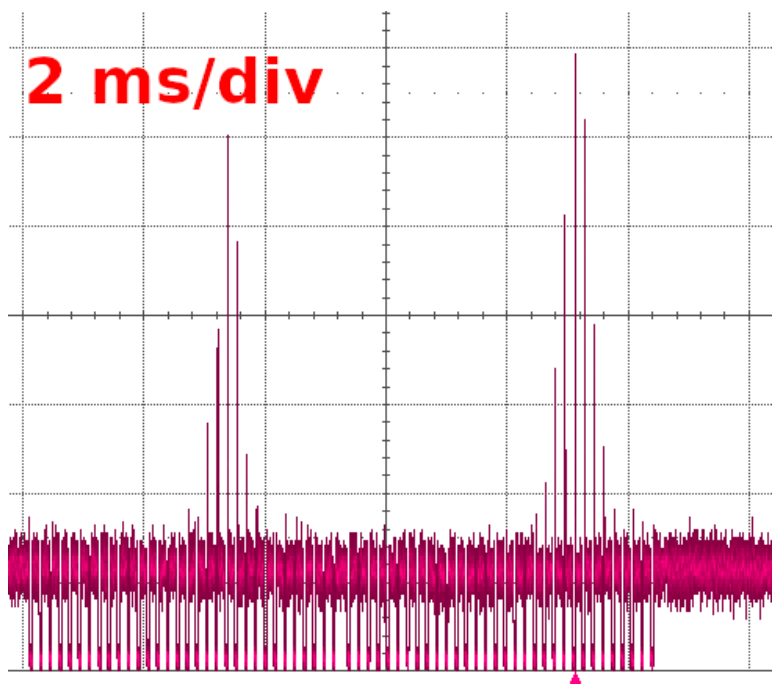

FIGURE 9. Image de la puissance observée par le détecteur (Analog Devices AD8362) d'un RADAR à courte portée à balayage de fréquence sondant la réponse d'un capteur différentiel présentant deux résonances (SENSeOR SEAS10). Un atténuateur de $42 \mathrm{~dB}$ est ici placé entre l'électronique de mesure et le capteur pour simuler les pertes de propagation (Free Space Propagation Loss) à une distance de l'ordre de $7 \mathrm{~m}$ en supposant le lecteur et le capteur munis d'antennes omnidirectionnelles. L'axe des abscisses de l'oscillogramme $(2 \mathrm{~ms} /$ division$)$ représente le balayage en fréquence de la source radiofréquence émise, l'ordonnée (200 mV/division) la tension image de la puissance reçue du capteur.

limitant : une liaison asynchrone à 115200 bauds limite une communication avec des trames de 10 caractères à un taux de rafraîchissement de $1152 \mathrm{~Hz}$, en-deça de la vitesse de mesure autorisée par la stratégie de mesure.

Cette méthode asservie, initialisée sur un balayage complet de la bande pour identifier les fréquences de résonance du capteur différentiel pour ensuite recaler la bande de mesure sur la fréquence acquise auparavant, présente par ailleurs le bénéfice de s'affranchir du biais introduit par l'anti-résonance : ce biais reste constant pour un facteur de qualité stable au cours des mesures.

\subsection{Méthode 2-points}

La dernière stratégie de mesure que nous détaillerons propose une approche inspirée des détections synchrones, dans lesquelles nous ne cherchons plus à identifier la fréquence de résonance mais uniquement à équilibrer la réponse du capteur sondé à deux fréquences de part et d'autre de la résonance [45]. Dans cette approche classique du convertisseur de modulation de fréquence en modulation d'amplitude par un filtre, le DDS est alternativement programmé pour sonder la puissance retournée par le capteur en-deçà et au-dessus de la résonance. Si la puissance reçue à ces deux 
fréquences n'est pas identique, un asservissement commande la fréquence moyenne de ces deux valeurs spectrales afin de suivre la résonance. Le principal gain de cette méthode, outre sa vitesse en réduisant le nombre de points de mesure et donc atteindre plus de 3000 mesures/seconde, est de convertir une mesure issue d'un ajustement polynomial du second ordre (parabole) en un asservissement sur une relation linéaire, plus stable et moins sensible aux bruits de mesure d'amplitude du signal reçu.

\section{PRINCIPE DE SENSIBILITÉ : GRANDEURS ACCESSIBLES}

\subsection{Propriété intrinsèque vs extrinsèque}

Le transducteur à ondes élastiques répond à deux exigences : séparer la réponse du capteur de l'ensemble des échos générés par l'environnement (clutter) et introduire une signature représentative de la grandeur mesurée (Fig. 2). L'approche que nous avons choisie d'exploiter pour distinguer le capteur de l'environnement consiste à retarder la réponse temporelle du premier au-delà de celle du dernier, et ce compte tenu de la sensibilité du récepteur. Ainsi, pour un récepteur ne présentant pas une sensibilité suffisante pour détecter une cible de grandes dimensions quelques centaines à milliers de mètres carrés de section efficace équivalente (voir encadré ci-dessous) - à plus de $150 \mathrm{~m}$, retarder la réponse du capteur de $1 \mu$ s suffit pour garantir que tout signal acquis après cette date est attribuable au capteur.

\section{Principes de conception}

L'argumentaire sur la section efficace RADAR équivalente d'une cible à une distance de $150 \mathrm{~m}$ qui renverrait un signal de la même puissance qu'un transducteur à onde élastique conçu comme cible coopérative se déroule comme tel :

1) l'équation du RADAR monostatique annonce une décroissance de la puissance reçue comme la puissance 4ème de la distance $d$ en supposant que la cible ponctuelle se comporte comme source d'une onde sphérique après intersection de la puissance incidente issue de l'émetteur,

2) la section RADAR équivalente $\sigma$ (en $\mathrm{m}^{2}$ ) est remplacée par les pertes d'insertion du transducteur $I L$ définies comme le rapport de la puissance incidente à la puissance rétrodiffusée, et par soucis d'homogénéité, nous remplaçons $\sigma$ par $\lambda^{2} . I L$ avec $\lambda$ la longueur d'onde électromagnétique

3) premier cas, un résonateur (décroissance de la puissance de $8,7 \mathrm{~dB} / \tau$ avec $\tau=Q /(\pi f)$ la constante de temps de décroissance du signal issu du résonateur de fréquence de résonance $f$ et de facteur de qualité $Q$ ) : à paramètres du bilan de liaison égaux (gain d'antennes), $I L \lambda^{2} / d_{S A W}^{4}=$ $\sigma / d_{R A D A R}^{4}$ avec $d_{S A W}$ la portée d'interrogation $\mathrm{du}$ résonateur et $d_{R A D A R}$ la distance à laquelle une cible de section équivalent $\sigma$ réfléchit la même puissance que le résonateur. Ainsi, $\sigma=$ $\left(\frac{d_{R A D A R}}{d_{S A W}}\right)^{2} \lambda^{2} . I L$. Supposons $d_{S A W}=5 \mathrm{~m}$ (antenne isotrope) et $d_{R A D A R}=150 \mathrm{~m}$ pour justifier du retard introduit dans la conception $\mathrm{du}$ capteur, alors pour un résonateur fonctionnant à $f=434 \mathrm{MHz}$ et $Q=10000$ (donc $\tau=7,3 \mu$ s et $I L=1 \mathrm{~dB}$ pour une mesure à $1 \mu$ s de l'émission), l'application numérique donne $\sigma=\lambda^{2} \times 800^{2}$ ou une cible carrée de $560 \mathrm{~m}$ de coté puisque $\lambda=70 \mathrm{~cm}$.

4) second cas, une ligne à retard (pertes d'insertion typiques de $30 \mathrm{~dB}$ dans la bande radiofréquence de $2400 \mathrm{MHz}$ pour laquelle $\lambda=$ $12,5 \mathrm{~cm}$ ), alors l'application numérique en prenant $d_{S A W}=1 \mathrm{~m}$ (cas d'une l'antenne isotrope) donne $\sigma=\lambda^{2} \times 700^{2}$ et nous n'avons plus qu'une cible carrée de $90 \mathrm{~m}$ de côté. Ces sections RADAR sont typiques des mesures de réflexions de batiments lors des mesures en RADAR passif [46].

5) de telles cibles limitent la capacité de détecter le signal d'un capteur à longue distance, mais le cas pathologique le plus dramatique est d'insérer un capteur dans une cavité électromagnétique résonante (autoclave, four) de facteur de qualité équivalent à celle du capteur. Dans ce cas, la mesure devient impossible, l'environnement électromagnétique éblouissant le RADAR courte portée servant à sonder la réponse du capteur.

Le rapport signal à bruit est ainsi excellent, puisque seul le bruit du récepteur pollue la mesure après un tel délai et un filtre adapté (inter-corrélation) fournit une excellente capacité de détection du signal du capteur. Le retard introduit par la cible coopérative doit d'une part retarder le signal suffisamment pour permettre au clutter de s'atténuer en-deçà de la puissance renvoyée par le capteur et ce malgré une section RADAR généralement bien plus importante que celle de ce dernier, et d'autre part limiter les pertes introduites par la propagation de l'onde élastique à la surface du substrat piézoélectrique. Ces dernières sont tabulées sous forme de $\mathrm{dB} / \mu \mathrm{s}$ dans [47, p.100] sous forme d'un développement au second ordre en fonction de 
la fréquence, pour atteindre de l'ordre de $5,5 \mathrm{~dB} / \mu \mathrm{s}$ à 2,4 GHz sur niobate de lithium (Fig. 10).

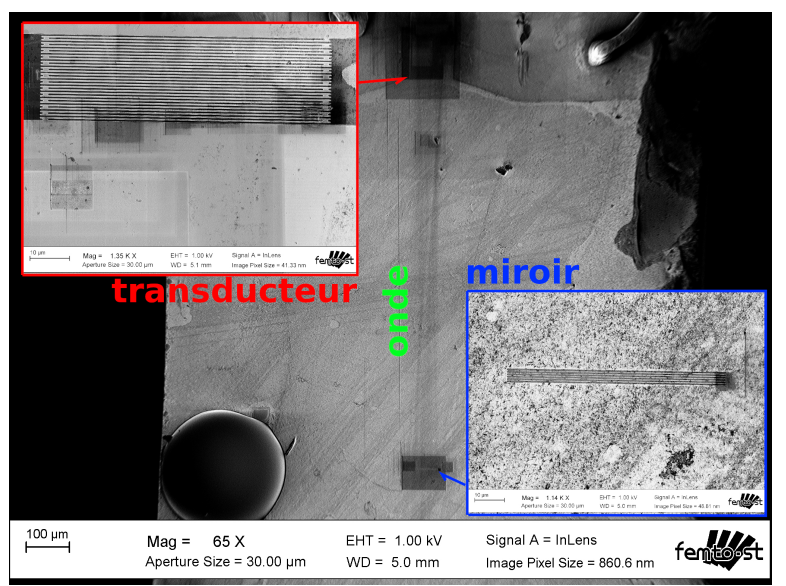

FIGURE 10. Image au microscope électronique à balayage d'une ligne à retard sur niobate de lithium fonctionnant à $2,4 \mathrm{GHz}$ avec une puissance d'excitation de $+15 \mathrm{dBm}$. La différence entre les dimensions des structures excitatrice (transducteur interdigité) et reflectrice (miroir) par rapport au chemin acoustique est mise en avant par les zooms en insert, ainsi que la collimation de l'onde visible le long du chemin acoustique.

Nous avons vu que les deux stratégies pour retarder le signal au-delà du bruit de l'environnement sont la ligne à retard ou le résonateur, dont le temps de décharge est proportionnel au ratio du facteur de qualité à la fréquence de résonance. Comment identifier la grandeur physique dans le signal retourné par le capteur? La physique du transducteur à ondes élastiques implique une variation de la vitesse de l'onde élastique sous l'effet de l'environnement du capteur. En effet, les coefficients électromécaniques du substrat piézoélectrique dépendent de paramètres tels que la température (Figs. 11) ou la contrainte [48]. À longueur d'onde constante, varier la vitesse de l'onde élastique se traduit par une variation de temps de vol (architecture de ligne à retard) ou une variation de fréquence de résonance (architecture de résonateur). La relation entre ces grandeurs mesurées et la grandeur physique impactant la vitesse de l'onde élastique se déduit en premier lieu par modélisation, sous réserve que les coefficients de sensibilité des coefficients électromécaniques du substrat piézoélectrique avec la grandeur mesurée soient identifiées et deuxièmement par étalonnage. En effet, la dispersion des paramètres de fabrication, notamment l'épaisseur de métal composant les électrodes, implique une dispersion des sensibilités qui nécessite un étalonnage individuel des capteurs. Une architecture de ligne à retard, dans laquelle l'onde élastique passe la majorité de son temps de vol en espace libre non-métallisée, présente une dispersion moindre que le résonateur dont la fréquence de résonance est notablement sensible aux propriétés géométriques des électrodes.

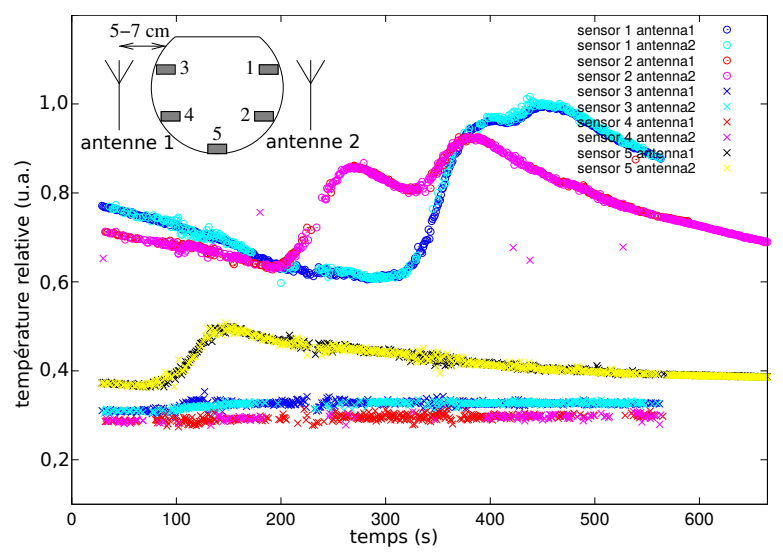

FIGURE 11. Exemple de mesure normalisée de température par cinq couples de résonateurs à ondes élastiques de surface placés en diverses positions radiales dans la gomme d'un pneu et mesurées en cours de roulage : le multiplexage fréquentiel permettant de différencier les sources des réponses des capteurs ne respecte pas la bande bande ISM. La vitesse de roulage au cours de ces mesures est de plus de $200 \mathrm{~km} / \mathrm{h}$.

Cette première architecture de capteur exploite la sensibilité intrinsèque du substrat piézoélectrique aux éléments paramétriques environnementaux. Une seconde approche consiste à exploiter une architecture de quadripôle dans laquelle une impédance variable avec la grandeur mesurée induit une variation de la vitesse de l'onde élastique. Une telle architecture extrinsèque présente cependant une faible sensibilité puisque l'impact de l'impédance sur les conditions de propagation de l'onde est un effet du second ordre. À titre d'exemple, la variation de vitesse de l'onde élastique entre des conditions de surface libre ou conductrice est proportionnelle au coefficient de couplage électromécanique. Une telle architecture exploitera donc favorablement un substrat fortement couplé tel que le niobate de lithium.

\subsection{Conception du capteur}

Un attrait des transducteurs à ondes élastiques face notamment aux dispositifs en silicium - est leur simplicité de réalisation en environnement de salle blanche : une unique étape de lithograhie/dépôt métallique suffit à définir les électrodes sur le substrat piézoélectrique qui, une fois polarisées par l'onde électromagnétique incidente, créeront l'onde élastique. Cependant, les degrés de liberté sur la conception du capteur restent nombreux, en particulier du fait de l'anisotropie du substrat monocristallin, la multitude de substrats, l'impact de la géométrie des électrodes 
(épaisseur, rapport de métallisation) et de leur motif. La conception de dispositifs à ondes élastiques a été développée en détail dans [49]. Le problème des capteurs est rendu plus ardu par la nécessité de composer avec l'impact de toutes les grandeurs physiques susceptibles d'interagir avec le substrat, non plus comme perturbations mais cette fois comme composantes dominant le comportement temporel ou spectral du dispositif. À cela, on rajoutera l'impact du conditionnement du capteur et son association à l'antenne pour les solutions sans fils qui participent quasiment au premier ordre de la fonction. Le problème est donc notablement plus compliqué que par exemple celui d'un filtre inséré dans un circuit RF.

Le dépôt d'électrodes interdigités au motif périodique induit une réponse limitée en bande passante. Cette bande passante est d'autant plus réduite que le nombre de doigts dans les peignes interdigités est important, une caractéristique exacerbée par l'architecture de résonateur qui confine l'onde dans la cavité acoustique définie par deux miroirs de Bragg déposés de part et d'autre du transducteur interdigité. Comme tout régime présentant une fréquence de fonctionnement propre, le comportement d'un dispositif à ondes élastiques de surface (ou de volume) peut être représenté par un schéma électrique équivalent de type RLC série appelé branche motionelle [50], [51]. Cependant, le dépôt des électrodes sur un substrat piézoélectrique donc en premier lieu diélectrique introduit une composante naturelle supplémentaire en parallèle de la branche motionelle, représentée par une capacité statique notée $C_{0}$ qui constitue donc la branche dite statique. Ce schéma équivalent à branches motionelle et statique, appelé Butterworth - Van-Dyke (BVD) - présente non seulement une résonance (maximum de courant dans la branche motionnelle formée du RLC série) mais aussi une anti-résonance, dans laquelle le courant dans la branche motionnelle est minimum et induit de ce fait un maximum d'impédance du circuit équivalent [51].

La prise en compte du comportement capacitif intrinsèque des transducteurs piézoélectriques et plus particulièrement à ondes élastiques tient une place centrale dans la conception des composants RF fondés sur ces principes. En effet, d'un point de vue électrique, l'association d'un tel composant avec une antenne afin de le rendre interrogeable à distance impose l'optimisation de leur affinité, soit un minimum d'accord d'impédance électrique. Si l'on prend comme exemple une antenne dipôle présentant une impédance de $75 \Omega$ à sa résonance, le capteur associé sera conçu pour présenter une impédance proche de cette valeur ou au moins comprise dans la gamme 50-100 $\Omega$ pour minimiser les pertes de transfert d'énergie entre les deux objets. Compte tenu de cette contrainte, le concepteur doit optimiser l'impédance de son capteur au voisinage des fréquences de travail. Cette optimisation est donc circonstanciée à la nature du substrat et à ses propriétés électriques intrinsèques. En clair, on ne conçoit pas un résonateur suivant des règles identiques selon qu'il soit sur quartz ou sur niobate de lithium, ne serait-ce que par la différence de propriétés diélectriques des deux matériaux (il existe un facteur 10 entre permittivités relatives respectives). D'un point de vue radioélectrique, ces considérations sont périphériques dans la mesure où l'important tient à l'accord d'impédance entre les composants du système. Or, grâce au phénomène d'anti-résonance, il existe deux possibilités pour le concepteur d'atteindre une impédance donnée, disons $50 \Omega$ pour simplifier, soit la résonance et l'antirésonance qui vont toutes deux correspondre à une contribution singulière, définie par une fréquence caractéristique et un étalement spectral associé à un coefficient de qualité. Ces deux points de fonctionnement vont se révéler plus ou moins favorables selon la permittivité diélectrique du substrat mais aussi selon la fonction spectrale recherchée. Pour les résonateurs, on démontre [52] qu'avec des matériaux tels que la langasite, le niobate ou le tantalate de lithium dont la permittivité diélectrique relative est comprise entre 30 et 50 , le choix de l'anti-résonance donne lieu à des dispositifs certes de dimensions notables mais compatibles avec les contraintes opérationnelles et peu sensibles aux éléments parasites tels que les résistances série introduites par la connectique. Il est bien entendu possible de choisir la condition de résonance pour de tels substrats mais il est alors impératif de croiser d'autres éléments constitutifs du composant avec cette première contrainte pour valider le fonctionnement final du capteur. En effet, compte tenu de la capacité statique induite par les propriétés diélectriques du substrat, le transducteur fonctionnant à la résonance présentera un nombre d'électrodes limité à quelques dizaines en considérant une ouverture acoustique de plus de 20 longueurs d'ondes (idéalement proche de 40). Ceci posé, nous introduisons un caractère critique du mode exploité, son couplage électromécanique. Par exemple, les substrats mentionnés plus haut donnent accès à des modes dont le couplage électromécanique s'étend de 0,1 à $6 \%$. Pour des couplages inférieurs à $0,5 \%$, la contribution piézoélectrique à la capacité statique constituée par les peignes inter-digités risque de donner lieu à une perturbation d'impédance très 
inférieure à celle escomptée, avec une variation de paramètre $S_{11}$ (absorption énergétique) inférieure à quelques $\mathrm{dB}$ et ainsi incompatible avec une interrogation efficace à distance de plus de la dizaine de centimètres. Pour les modes à couplage supérieur au pourcent, la sensibilité aux éléments de connectique empêche en pratique de tirer parti des caractéristiques intrinsèques du résonateur. On démontre que le choix de l'anti-résonance dans ces cas de figure est toujours plus favorable électriquement, certes au prix d'une dimension notablement plus importante qu'à la résonance (un facteur 10 en surface). Par contre, choisir l'anti-résonance pour une ligne à retard n'est pas souhaitable dans la mesure où ce point de fonctionnement ne maximise pas la conversion acoustoélectrique, cette fonction étant naturellement obtenue à la résonance. En effet, le principe de la ligne à retard réflective est fondé sur l'émission d'une onde élastique suffisamment énergétique pour parcourir plusieurs centaines de longueurs d'onde entre de multiples réflecteurs et donner lieu à une réémission par le transducteur détectable par l'interrogateur. il faut donc choisir un point de fonctionnement maximisant le couplage électromécanique. La notion de résonance peut alors sembler inappropriée sachant qu'une ligne à retard présente une caractéristique physique large bande, au contraire de celle d'un résonateur à bande dite étroite. Nous généralisons notre propos en parlant alors de maximum de conductance pour la condition de résonance et de maximum de résistance pour celle de l'antirésonance. Pour finir sur cette question, nous noterons que des dispositifs fonctionnant à la résonance sont naturellement adaptés à une association en circuit parallèle alors que des dispositifs fonctionnant à l'antirésonance seront avantageusement couplés en montage série [52].

\subsection{Conception du capteur : choix du substat}

La difficulté de conception des capteurs, notamment par rapport aux résonateurs pour oscillateurs ultra-stables ou encore aux filtres de fréquence, tient à la grande variabilité de l'environnement auquel le corps d'épreuve est soumis. La conception du capteur consiste donc à optimiser la sensibilité du corps d'épreuve généralement constitué autour du/des résonateur(s) ou de la ligne à retard à la grandeur mesurée, tout en réduisant au maximum l'impact des grandeurs interférant sur la mesure. Une telle conception passe nécessairement par une étape de modélisation prenant en compte les effets croisés des diverses grandeurs physiques sur la vitesse de phase de l'onde élastique au premier ordre et donc sur le déphasage ou la fréquence de résonance mesurées mais aussi de façon globale sur l'ensemble des propriétés physiques du capteur potentiellement affectées par les variations environnementales. Comme partiellement évoqué dans le chapitre précédent, le choix du substrat ne se limite pas au choix du mode et de ses propriétés acoustiques, ni à l'alternative ligne à retard-résonateur. En effet, un tel critère pourrait se déduire d'une simple analyse, pour les résonateurs, du facteur de qualité $Q$ au point de fonctionnement choisi (résonance/antirésonance, cf. chapitre précédent). Fondé sur l'étude de [15], la résistance introduite par les transducteurs interdigités réduit d'autant plus le facteur de qualité que le courant qui y circule est important. Le choix d'un substrat à couplage électromécanique élevé (>1\%) se révélerait ainsi inadapté à l'application. Toutefois, la pertinence de ce choix va essentiellement dépendre de la condition de fonctionnement retenue, comme nous l'avons vu plus haut. En particulier, sélectionner l'antirésonance pour de tels matériaux s'avère compatible avec l'obtention de paramètres fonctionnels exploitables pour la mesure. Pour les lignes à retard, se focaliser exclusivement sur le couplage ne permet pas de garantir une dynamique de réponse maximale pour un nombre de pics donnés sur la réponse impulsionnelle. De même, maximiser ce critère est insuffisant pour équilibrer les pics de ladite réponse, garant d'un détection homogène et uniforme pour des conditions d'interrogation optimale du capteur.

Pour les résonateurs, un subtil compromis existe entre le niveau de puissance initial restitué par le capteur, proportionnel à la puissance incidente multipliée par le coefficient de couplage $k_{s}^{2}$ et la décroissance exponentielle de la réponse avec une constante de temps $Q /\left(\pi f_{0}\right)$ : si $Q$ est inversement proportionnel à $k_{s}^{2}$, le compromis peut alors balancer en faveur d'un $Q$ médiocre (correspondant à un produit $Q \times f \mathrm{de}$ l'ordre de $10^{12}$ ) permettant au signal restitué par le capteur de dépasser le niveau de bruit du récepteur et des réflecteurs environnants, plutôt que de maintenir un signal faible pendant une très longue durée tel qu'observé avec un $Q$ élevé mais un $k_{s}^{2}$ faible $(<1 \%$, cas du substrat de quartz). Rappelons en effet que l'objectif est uniquement de maintenir une réponse à environ $1 \mu \mathrm{s}$ de l'émission de l'impulsion sonde et qu'un facteur de qualité de plus de 3000 à $434 \mathrm{MHz}$ (soit $\tau=2 \mu$ s) n'a aucun intérêt pour la mesure autre que de ralentir la vitesse d'interrogation pour garantir l'indépendance des mesures successives. Le dilemme devient d'autant plus grand que les substrats à couplage électromécanique supérieur à $1 \%$ comme les tantalate et niobate de lithium présentent une permittivité relative effective [47, p.75] $\varepsilon_{e f f}$, décrivant 
le coefficient diélectrique équivalent accompagnant le champ électrique entre deux électrodes dans la direction de propagation, (tableau I) telle que l'impédance à la résonance, pour un transducteur donné, tend vers des valeurs nettement inférieures à $50 \Omega$ à la résonance compte tenu de la capacité statique ainsi induite, comme exposé auparavant. Pour un matériau présentant une résistivité de l'ordre de $100 \mathrm{G} \Omega . \mathrm{cm}$, le choix de l'anti-résonance comme point de fonctionnement permet de minimiser la sensibilité du dispositif aux éléments électriques introduits par la connectique (résistance de contact en série avec le composant), de maximiser le coefficient de qualité en tirant au maximum parti des propriétés acoustiques du substrat sans que d'autres sources de pertes viennent dégrader la réponse du dispositif.

Pour les lignes à retard, il est tout d'abord nécessaire d'utiliser un vrai mode puisque la propagation y joue un rôle majeur. Ce critère élimine d'emblée les pseudo ondes de surface (leaky-SAW en anglais) et donc un grand nombre de configurations potentiellement avantageuses sur niobate et tantalate de lithium compte tenu du couplage électromécanique des ondes correspondantes. Toutefois, si le coefficient de couplage joue un rôle premier dans la dynamique de la réponse impulsionnelle d'une ligne à retard, il faut également tenir compte des pertes de propagation composées des pertes intrinsèques du substrat mais aussi des effets de diffraction (pour un retard de l'ordre de la $\mu$ s à 2,45 GHz, les ondes peuvent se propager sur plusieurs centaines de longueurs d'onde), de possibles couplages physiques (rayonnement dans le milieu adjacent d'autant plus faible cependant que la fréquence de travail est élevée) et du coefficient de réflexion sur obstacle unique. Choisir pour ce dernier une valeur supérieure à $10 \%$ n'a de sens que pour une ligne à retard à nombre d'échos ne dépassant pas 3. En effet, une telle intensité de réflexion rend la conception de lignes à retard à plus de 3 échos très délicate, en particulier pour garantir un niveau d'amplitude équilibré entre chaque contribution. On voit donc que là encore, tout est affaire de compromis et de cahier des charges.

Tout au long de cet exposé, nous avons mis en évidence une dualité entre les dispositifs large bande que sont les lignes à retard et les dispositifs à bande étroite que sont les résonateurs. Une architecture de transducteurs est attractive pour les mesures sans fil de grandeurs physiques car elle présente simultanément ces deux caractéristiques, favorisées selon le mode d'interrogation sélectionné : les transducteurs à ondes de volume d'harmonique élevée (High-overtone Bulk Acoustic Resonator - HBAR). Ces dispositifs
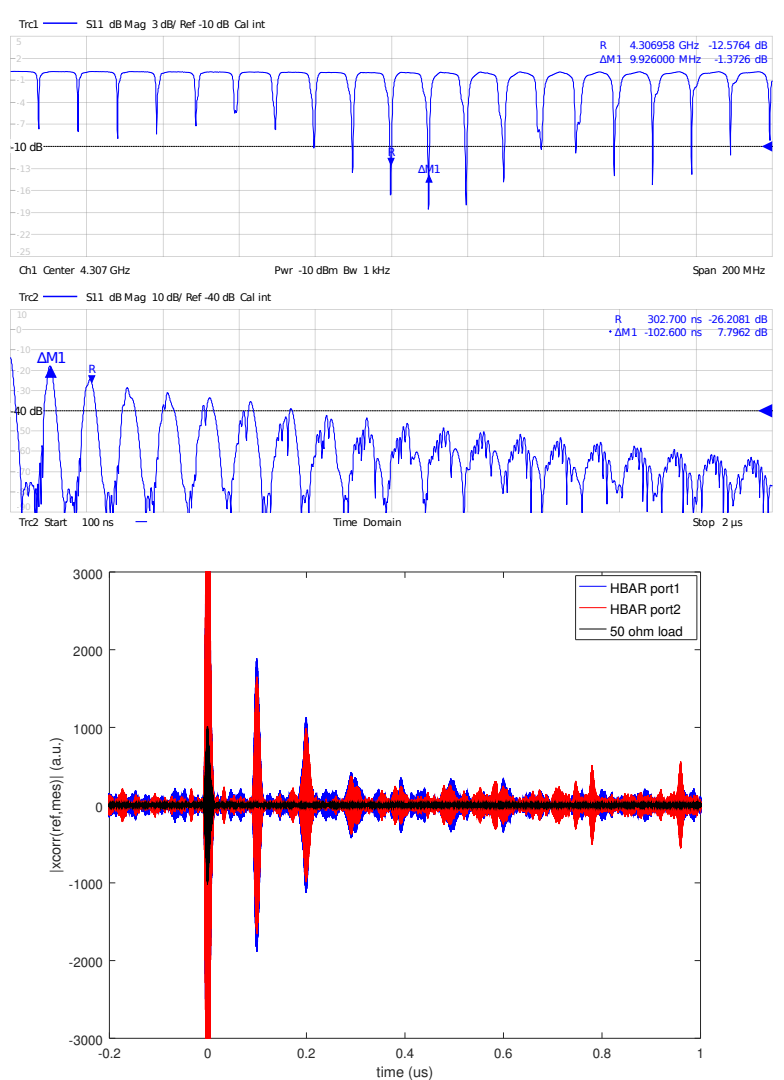

FIGURE 12. Haut : caractérisation spectrale (haut) et temporelle (bas) d'un résonateur HBAR sur une plage de fréquences autour de 4,3 GHz (bande de fréquence allouée à WAIC). Bas : mesure de HBAR par un RADAR à bruit : le capteur est illuminé par une porteuse à $4,3 \mathrm{GHz}$ modulée en phase par une séquence pseudoaléatoire à $150 \mathrm{Mb} / \mathrm{s}$. L'intercorrélation du signal reçu du capteur avec la séquence émise permet de retrouver le retard introduit par le HBAR appréhendé comme ligne à retard.

sont formés d'une couche piézoélectrique pour laquelle la fréquence du mode de volume fondamental (de cisaillement ou longitudinal selon la configuration cristalline retenue) se situe entre quelques centaines de $\mathrm{MHz}$ et plusieurs $\mathrm{GHz}$ placée (dépôt en phase vapeur ou par report-amincissement) sur un substrat d'épaisseur 10 à 100 fois supérieure présentant des pertes intrinsèques minimales (saphir, quartz, silicium haute résistivité et qualité structurelle, tantalate ou niobate de lithium, YaG, YiG, etc.). La couche piézoélectrique pompe, par couplage électromécanique, de la puissance dans le substrat qui détermine le facteur de qualité de chaque mode. Le rapport des épaisseurs de la couche piézoélectrique $e_{p}$ au substrat $e_{s}$ détermine l'ordre de grandeur du mode excité dans ce dernier, typiquement $e_{s} / e_{p}$ de plusieurs dizaines à quelques centaines, déterminant le 
TABLE I

PRINCIPAUX MATÉRIAUX UTILISÉS POUR LA RÉALISATION DE TRANSDUCTEURS À ONDES ÉLASTIQUES : LES SUBSTRATS PRÉSENTANT DE FORTS COEFFICIENTS DE COUPLAGE ÉLECTROMÉCANIQUES $\left(k_{s}^{2}\right)$ SONT PLUS FAVORABLES POUR LES LIGNES À RETARD, LES MATÉRIAUX FAIBLEMENT COUPLÉS (QUARTZ) POUR LES RÉSONATEURS SOUS RÉSERVE DES CONTRAINTES DE CONCEPTION MENTIONNÉES EN SECTION 5.3 LES CALCULS DE VITESSE, COUPLAGE ÉLECTROMÉCANIQUE, COEFFICIENT DE RÉFLEXION ET COEFFICIENT EN TEMPÉRATURE DE LA FRÉQUENCE (CTF) SONT FAITS POUR UNE ÉPAISSEUR D'ALUMINIUM DE $h / \lambda=2 \%$ ET UN RAPPORT DE MÉTALLISATION À LA PÉRIODE DU TRANSDUCTEUR DE $a / p=0,5$.

\begin{tabular}{|c|c|c|c|c|c|c|c|}
\hline Substrat & $\begin{array}{c}\text { Coupe (Yxwlt) } \\
\phi / \vartheta / \psi\left({ }^{\circ}\right)\end{array}$ & Type d'onde & $\begin{array}{l}\text { vitesse } \\
(\mathrm{m} / \mathrm{s})\end{array}$ & $k_{s}^{2}(\%)$ & $\begin{array}{l}\text { coefficient de } \\
\text { réflexion/électrode }(\%)\end{array}$ & $\varepsilon_{e f f}$ & $\begin{array}{l}\text { CTF 1er ordre }\left(\mathrm{ppm} . \mathrm{K}^{-1}\right) \\
(\mathrm{CTF} 2 \text { si } \mathrm{CTF} 1 \simeq 0)\end{array}$ \\
\hline \multirow[t]{4}{*}{ quartz } & $0 / 34 / 0$ & Rayleigh & 3133,58 & 0,116 & 1,21 & 4,51 & $0\left(-32\right.$ ppb.K $\left.\mathrm{K}^{-2}\right)$ \\
\hline & $0 / 36 / 0$ & Rayleigh & 3137,54 & 0,118 & 1,22 & 4,51 & $0\left(-37\right.$ ppb. $\left.\mathrm{K}^{-2}\right)$ \\
\hline & $0 / 42,75 / 0$ & Rayleigh & 3143,95 & 0,119 & 1,14 & 4,52 & $0\left(-42 \mathrm{ppb} . \mathrm{K}^{-2}\right)$ \\
\hline & $0 / 36 / 90$ & STW & 5053,78 & 0,216 & 1,36 & 4,56 & $0\left(-55 \mathrm{ppb} . \mathrm{K}^{-2}\right)$ \\
\hline \multirow[t]{3}{*}{$\mathrm{LiTaO}_{3}$} & $-90 / 0 / 112$ & Rayleigh & 3284,60 & 0,766 & 1,25 & 47,39 & -18 \\
\hline & $0 / 36 / 0$ & PSAW & 4136,89 & 6,40 & 3,98 & 49,26 & -35 \\
\hline & $0 / 42 / 0$ & PSAW & 4140,90 & 6,06 & 3,80 & 48,83 & -35 \\
\hline \multirow[t]{4}{*}{$\mathrm{LiNbO}_{3}$} & $0 / 0 / 90$ & Rayleigh & 3421,61 & 4,43 & 1,96 & 44,98 & -94 \\
\hline & $0 / 128 / 0$ & Rayleigh & 3878,37 & 5,70 & 0,8 & 54,70 & -74 \\
\hline & $0 / 64 / 0$ & PSAW & 4523,90 & 11,54 & 6,77 & 50,93 & -72 \\
\hline & $0 / 41 / 0$ & PSAW & 4472,87 & 19,08 & 10,0 & 60,94 & -52 \\
\hline \multirow[t]{2}{*}{ Langasite } & $0 / 48.5 / 26.6$ & Rayleigh & 2735,82 & 0,23 & 1,04 & 27,97 & $0\left(-60 \mathrm{ppb} \cdot \mathrm{K}^{-2}\right)$ \\
\hline & $\mathrm{YX}$ & Rayleigh & 2320 & 0,38 & 0,15 & 20,4 & 38,5 \\
\hline $\mathrm{Li}_{2} \mathrm{~B}_{4} \mathrm{O}_{7}$ & $0 / 45 / 90$ & Rayleigh & 3401,0 & 1,0 & 7,0 & 9,6 & $0\left(-270\right.$ ppb.K $\left.\mathrm{K}^{-2}\right)$ \\
\hline
\end{tabular}

nom de cette architecture de transducteur. Les modes successifs sont séparés spectralement de $\Delta f$ par les conditions aux limites de confinement de l'onde dans le substrat épais : si la vitesse de l'onde dans le substrat est $v_{s}$, alors $\Delta f=v_{s} /\left(2 e_{s}\right)$, ou pour $v_{s} \simeq 5000 \mathrm{~m} . \mathrm{s}^{-1}$ et $e_{s}=500 \mu \mathrm{m}$ en négligeant la contribution de la couche mince piézoélectrique dans le temps de vol de l'onde, un espacement spectral des modes de $\Delta f=5 \mathrm{MHz}$. Par dualité des réponses temporelles et spectrales, le peigne spectral de mode peut aussi être considéré comme un peigne temporel d'échos séparés d'un retard de $1 / \Delta f=200 \mathrm{~ns}$, parfaitement adapté pour l'application de cibles coopératives pour une mesure sans fil (Fig. 12). Le dernier critère de conception tient donc dans le coefficient de couplage électromécanique de chaque mode. Le coefficient de couplage électromécanique intrinsèque du substrat piézoélectrique de la couche pompe décroît comme le carré du numéro de l'harmonique de la couche mince qui pompe la puissance dans le substrat et ce coefficient de couplage est distribué entre les modes compris dans l'enveloppe de l'harmonique des modes pompés [16] : ainsi, un compromis sur l'épaisseur du subtrat supportant la couche piézoélectrique est d'être suffisamment fine pour réduire le nombre de modes sur lesquels se distribue le coefficient de couplage électromécanique du mode-pompe fondamental de la couche piézoélectrique - réduisant de ce fait le coefficient de couplage de chaque mode - mais suffisamment épais pour que les échos successifs restent détectables par l'électronique de mesure. Pour un délai de l'ordre de 500 ns qui reste acceptable pour le RADAR qui interroge le capteur, alors un substrat de 1,25 mm d'épaisseur serait idéal. Compte tenu des standards en vigueur pour les plaques monocristallines destinées aux applications de la microélectronique (250 $\mu \mathrm{m}-1 \mathrm{~mm})$, cette contrainte s'avère quelque peu limite face aux réalisations actuelles.

À titre d'illustration des perspectives offertes par le résonateur HBAR, notamment pour la montée en fréquence et appréhender les bandes spectrales audessus de $3 \mathrm{GHz}$, la Fig. 12 présente une interrogation sans fil dans la bande WAIC de 4,3 GHz allouée pour l'instrumentation par réseaux de capteurs d'avions [53]. Aucune perspective de commercialisation de HBAR pour des applications capteur ne semble néanmoins envisagée à la date de rédaction de ce document.

\section{EXEMPLES D’APPLICATION}

Divers exemples d'applications sont illustrés dans cette section. La sélection, arbitraire, d'applications vise à mettre en évidence les caractéristiques uniques des transducteurs à ondes élastiques utilisés comme cible coopérative de RADAR à courte portée. Nous noterons par exemple la capacité à encapsuler le capteur dans un matériau qui ne permettra plus d'accéder à la source d'énergie qui alimenterait un dispositif actif (gomme de pneu pour la mesure de température, pression [54], [55] ou contrainte [56], [57], béton), l'installation sur un objet mobile avec une vitesse de rotation élevée (moteur électrique de puissance, avec une rotation à plus de 5000 tours/minute) ou 
température supérieure à la gamme de fonctionnement des dispositifs en silicium $\left(800^{\circ} \mathrm{C}\right)$.
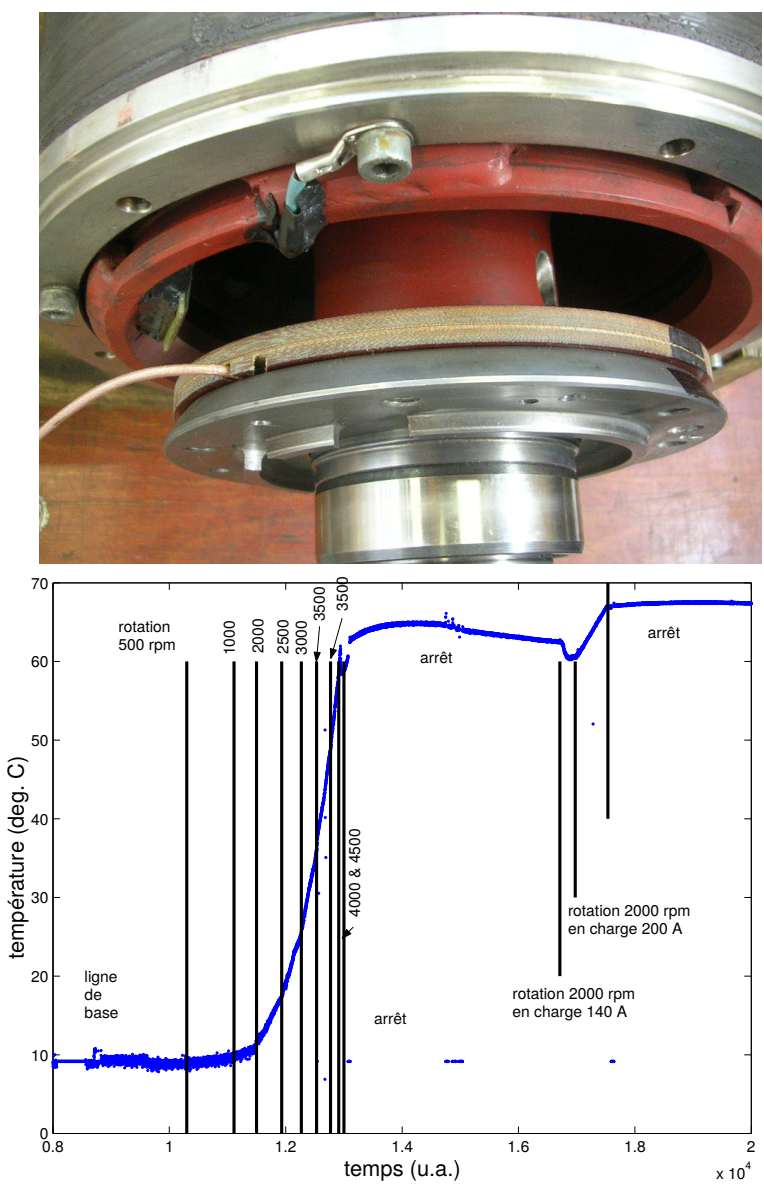

FIGURE 13. Mesure de température de moteur électrique de puissance dont un roulement est défectueux, induisant un échauffement excessif. Haut : montage d'un capteur sur le rotor d'un moteur électrique avec l'antenne reliée au RADAR fixée sur le stator selon une configuration de dipôle couvrant environ $120^{\circ}$ de la circonférence. Bas : mesure de la température du rotor pour des vitesses de rotation allant jusqu'à 4500 tours/minute.

L'instrumentation des rotors de moteur et turbine est un domaine d'application particulièrement approprié pour les capteurs passifs interrogeables sans fil. Nous avons déjà mentionné (Fig. 11) l'instrumentation de pneumatiques en insérant des résonateurs dans la gomme. La Fig. 13 illustre un autre exemple de mesure de température sur un rotor dont le roulement est défectueux, induisant un échauffement excessif représentatif du dysfonctionnement. Dans ce cas, une couverture angulaire de $90^{\circ}$, imposée par l'encombrement réduit de l'antenne sur le rotor, ne permet une durée de visibilité du capteur par le lecteur que de $85 \mu$ s. Une telle durée est cependant suffisante pour sonder 3 points d'un résonateur et en extraire la fréquence de résonance (Fig. 13). Une alternative aux stratégies de mesure rapide de résonateur repose sur la conception d'une antenne de couverture angulaire égale à $360^{\circ}$ [58]. L'instrumentation de parties mobiles de pièces d'usinage requiert de répondre à des exigences similaires [59]. Transense s'est ainsi spécialisé dans la mesure de couple sur arbre en rotation (moteurs, éoliennes) en installant deux capteurs de contrainte sur une géométrie appropriée pour extraire le couple à partir de deux jauges de contraintes orthogonales [60]. On notera cependant que l'utilisation des transducteurs à ondes élastiques comme capteur de contrainte est très spécifique et ne s'apparente pas à celle des jauges extensométriques résistives [61]. Les adhésifs organiques classiquement utilisés avec les jauges de déformation résistives souples ne peuvent être utilisés avec des substrats piézoélectriques monocristallins rigides que pour des contraintes inférieures à leur limite de plasticité, soit quelques dizaines de MPa pour les plus rigides. Au-delà de ce régime, le transfert des contraintes au capteur cristallin n'est plus garanti. Par ailleurs, au voisinage de cette limite, la linéarité du capteur est notablement dégradée, on ne peut donc espérer une mesure de contrainte fiable par ce moyen au-delà d'une trentaine de MPa sur acier et d'une quinzaine de MPa sur aluminium. À titre d'illustration d'une mesure de contrainte dans un environnement qui ne saurait être appréhendé autrement que par des capteurs passifs compte tenu de l'incapacité d'y accéder après déploiement, les tuyaux enterrés (distribution de gaz, produits pétroliers et autres fluides) nécessitent l'identification et la mesure de pression au travers de la contrainte pour identifier tout risque de rupture : un tel dispositif et son environnement de test sont présentés en Fig. 14

La nature et l'épaisseur du film de colle liant le corps d'épreuve au capteur de contrainte à onde élastique impactent considérablement la capacité de transfert de la contrainte. Dans le cas des corps d'épreuve rigide, des solutions de report par diffusion de film métallique sont explorées pour fournir un lien rigide entre corps d'épreuve et capteur non déformable. Dans le cas des corps d'épreuve souples (e.g. matériau composite avec une matrice organique ou gomme de pneu), le capteur influe signficativement sur le champ de contrainte. Une démonstration de mesure vibratoire, avec un résonateur à onde élastique soudé par brasure plomb-étain à la base d'un diapason musical (fréquence propre nominale de $440 \mathrm{~Hz}$ ), est illustrée en Fig. 15, mettant en évidence la vitesse de rafraîchissement de la mesure par la méthode d'interrogation 2-points développée auparavant (section 4.3. 

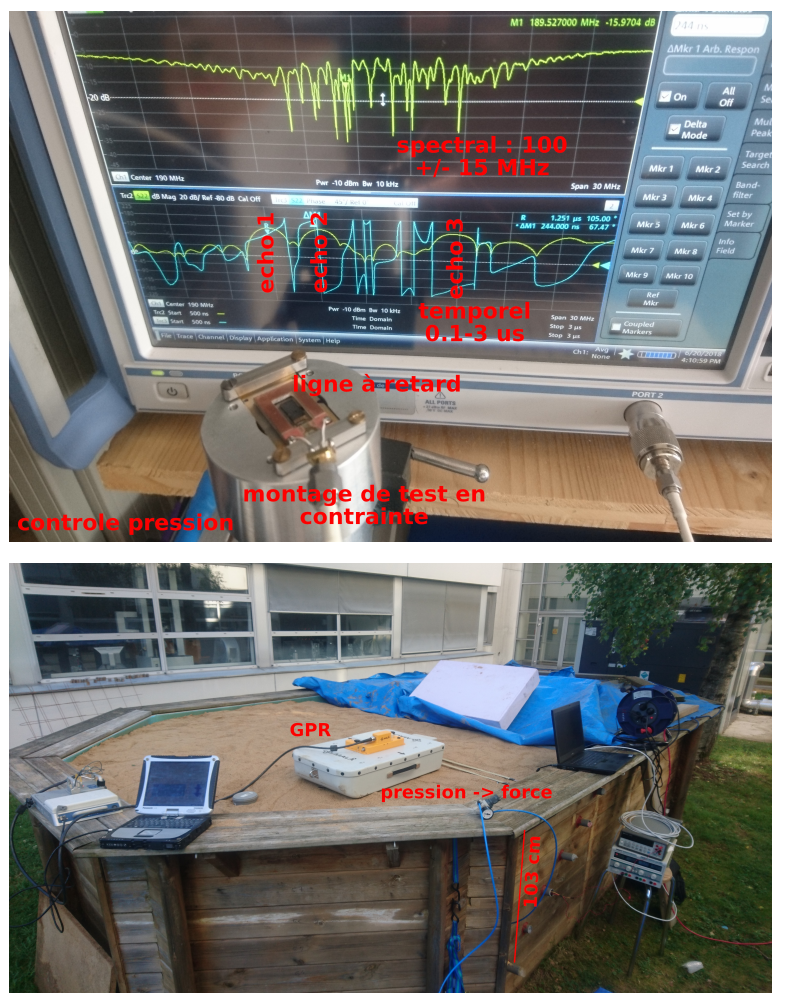

FIGURE 14. Haut : ligne à retard réflective conçue pour fonctionner dans la bande $100 \pm 15 \mathrm{MHz}$ comme cible coopérative d'un RADAR de sol, montée comme capteur de contrainte sur un corps d'épreuve en acier inoxydable. Le montage de qualification visible devant l'analyseur de réseau (en haut la réponse spectrale, en bas temporelle par transformée de Fourier inverse) applique une force sur un montage 3-points en variant la pression dans un piston sans affecter le bilan de liaison radiofréquence par l'insertion de fils conducteurs entre le capteur et son environnement. Bas : environnement expérimental de qualification du capteur de contrainte pour des mesures sub-surfaces (instrumentation de tuyaux enterrés) permettant des mesures par RADAR de sol dans le sable ou le gravier de capteurs enterrés jusqu'à $1 \mathrm{~m}$ de profondeur.

Cet algorithme de mesure a été utilisé dans [59] pour caractériser la contrainte, pendant la découpe, dans une dent de fraiseuse instrumentée.

Les méthodes de mesure rapide de la réponse de transducteurs sont applicables non seulement sur des objets en rotation présentant une visibilité intermittente de l'antenne du système d'interrogation mais aussi lorsqu'une bande passante importante de mesure est requise, par exemple pour une mesure de vibration à l'aide d'un capteur de contrainte. Dans le cas des résonateurs, le facteur de qualité limite la fréquence de rafraîchissement en imposant d'attendre la décharge du résonateur avant de solliciter le capteur avec un nouveau signal radiofréquence. Un compromis sur le facteur de qualité entre séparation de la réponse du capteur de son environnement (fort facteur de qualité,

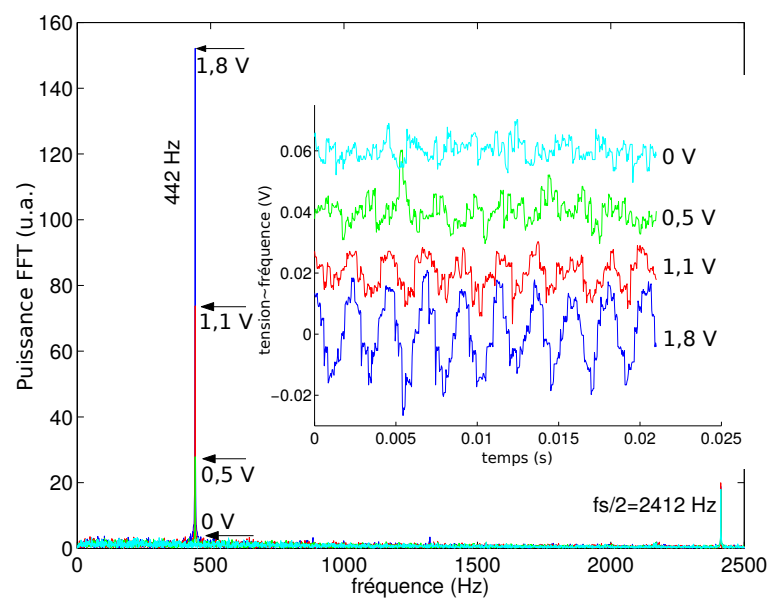

FIgURE 15. Analyse spectrale de la séquence de mesures de fréquences de résonance d'un résonateur à ondes de surface exploité comme capteur de contrainte, soudé à la base d'un diapason musical en acier inoxidable. La demi-fréquence de rafraîchissement est visible comme une raie à $2412 \mathrm{~Hz}$. En insert, les mesures temporelles traitées pour fournir les spectres : les tensions indiquent l'amplitude du signal alimentant un microphone dont le solénoïde entretient la vibration du diapason.

$Q>8000$ ) et taux de rafraîchissement élevé (faible facteur de qualité, $Q<4000$ ) permet de sélectionner l'architecture du capteur en fonction du critère à maximiser. À titre d'exemple, la caractérisation des modes de vibration d'un diapason musical (fondamental à $440 \mathrm{~Hz}$ ) a pu se faire par interrogation d'un résonateur fixé à la base d'un bras par une stratégie de mesure ne nécessitant que deux points de mesure, soit un taux de rafraîchissement de l'ordre de $2 \times 2 Q /(\pi f)$ avec $f$ la fréquence de résonance du capteur. Si nous choisissons $Q=3000$ à $f=434 \mathrm{MHz}$, alors la bande passante de mesure est de l'ordre de $100 \mathrm{kHz}$. En pratique, en attendant cinq constantes de temps pour totalement décharger le résonateur, une bande passante de mesure de l'ordre de $20 \mathrm{kHz}$ est accessible via un résonateur [48]. Une telle vitesse de mesure reste largement en-deçà des stratégies impulsionnelles de lignes à retard - dispositifs de bande passante importante - qui ne sont limitées que par la durée de l'écho le plus long, typiquement de l'ordre de $2 \mu$ s permettant donc une fréquence de rafraîchissement de l'information de $500 \mathrm{kHz}$ [29].

L'utilisation de capteurs à ondes élastiques de surface pour la mesure de températures supérieures à celles accessibles aux capteurs actifs ou à jonctions semi-conductrices sur silicium $\left(>300^{\circ} \mathrm{C}\right)$ constitue un domaine d'application attractif, tout en restant interrogeable sans fil. Des mesures à plus de $700^{\circ} \mathrm{C}$ ont ainsi été démontrées [62], [63], [64] en utili- 
sant comme substrat piézoélectrique des matériaux de synthèse tels que langasite et langatate [65] : ces matériaux présentent des effets piézoélectriques quasiment jusqu'à leur température de fusion [66] Toutefois, si l'utilisation de la langasite comme substrat pour capteur de température s'avère exploitable jusqu'à $650^{\circ} \mathrm{C}$ environ, l'accroissement de sa conductivité électrique au-delà de cette température le rend peu attractif pour des valeurs supérieures à la limite prescrite plus haut. Par ailleurs, au delà de $700^{\circ} \mathrm{C}$, des précautions sont à prendre quant à l'intégrité des électrodes dont le matériau est généralement choisi pour sa température de fusion supérieure à $1500^{\circ} \mathrm{C}$ (Ti/Pt, Ta/Pt, Ir, Zr, Mo et de composés à base de ces matériaux par exemple). Une passivation de surface par dépôt conforme d'oxydes $\left(\mathrm{SiO}_{2}, \mathrm{Al}_{2} \mathrm{O}_{3}\right)$ ou de couches nitrurées $\left(\mathrm{Si}_{3} \mathrm{~N}_{4}, \mathrm{AlN}\right)$ s'avère nécessaire pour des durées d'exploitation supérieures à 500 heures au-delà de $600^{\circ} \mathrm{C}$. Si des applications industrielles commencent à voir le jour pour des températures jusqu'à $650^{\circ} \mathrm{C}$, atteindre les $1000^{\circ} \mathrm{C}$ de façon fiable et répétable reste encore du domaine de la recherche.

\section{CONClusion}

En complément de l'utilisation de RADARs pour sonder la vitesse ou la position de cibles (niveau de fluides, vitesse de projectiles), nous avons présenté les règles de conception de cibles coopératives dont diverses propriétés de la section RADAR traduisent une grandeur physique. Les cibles passive coopératives fournissent une solution originale pour des environnements complexes qui ne présentent pas d'alternative (haute température, capteur inaccessible après installation - pneus, béton, sous-terre - ou en rotation/mouvement). Leur déploiement complexe compte tenu de la liaison RADAR qui nécessite de maîtriser le bilan de liaison entre l'électronique d'interrogation et le capteur impose de comprendre en détail la chaîne de mesure complète. Nous avons donc abordé les aspects spécifiques à cette liaison, incluant les architectures des électroniques d'interrogation s'apparentant aux RADARs à courte portée, algorithme de traitement des signaux acquis, conception du capteur selon les deux grandes archictures que sont les lignes à retard et les résonateurs et finalement fourni quelques exemples d'applications.

\section{GLOSSAIRE}

Bruit (noise)

signal dont l'auto-corrélation ne présente une valeur non-négligeable que pour un retard nul. En présence d'échos retardés dans le temps, par exemple par une ligne à retard à onde élastique, de multiples pics de corrélation sont représentatifs de ces retards. Le RADAR passif exploitant des sources noncoopératives exploite la statistique s'apparentant au bruit de ces signaux pour sonder la réponse de cibles coopératives à ondes élastiques

Cible coopérative (cooperative target)

transducteur donc la fonction de transfert spectrale (gamme fréquences de fonctionnement) et temporelle (retard des échoes) est concuse pour correspondre aux propriétés spectrales et temporelles du RADAR utilisé pour les interroger.

\section{Facteur de qualité (quality factor)}

capacité d'un résonateur à emmagasiner de l'énergie - ici sous forme d'onde élastique confinée dans une cavité entre deux mirroirs de Bragg - et la restituer selon une constante de temps de $Q / \pi$ périodes de l'onde.

\section{HBAR (High-overtone Bulk Acoustic Resonator)}

architecture de résonateur à onde de volume permettant une utilisation soit comme résonateur présentant une multitude de modes, soit comme ligne à retard présentant une multitude d'échos

\section{Ligne à retard (reflective delay line)}

architecture de dispositif à onde de surface dans laquelle une onde est générée par un tranducteur interdigité, se propage en espace libre jusqu'à rencontrer un ou plusieurs mirroir de Bragg, pour être réfléchie une unique fois et re-émise sous forme d'onde électromagnétique.

Onde de surface (surface acoustic wave) : onde élastique confinée à la surface d'un solide. Technologiquement, la génération sur substrat piézoélectrique par dépôt d'électrodes interdigitées est apparue comme une solution optimale en terme de compacité et efficacité de conversion électromécanique.

\section{Résonateur (resonator)}

architecture de dispositif à onde de surface dans laquelle une onde est générée par un tranducteur interdigité, se propoage jusqu'à rencontrer un mirroir de Bragg, pour être réfléchie une mutitude de fois dans la cavité ainsi formée, le nombre de trajets dans la cavité définissant le facteur de qualité.

Transducteur interdigité (interdigitated trasducer) : architecture d'électrodes se présentant comme deux peignes imbriqués permettant de générer un champ électrique dans le substrat piézoélectrique en vue de sa conversion en déformation mécanique. La déformation mécanique périodique se propage sous 
forme d'onde.

RADAR $(R A D A R)$

émetteur-récepteur d'onde électromagnétique.

La même antenne sert à émettre et recevoir

dans une architecture monostatique, tandis que antenne émettrice et réceptrice sont distinctes dans une architecture bi-statique. Parmi les RADARs classiquement utilisés, les RADARs de sol (Ground Penetrating RADAR - GPR) sont utilisés pour sonder la réponse de capteurs enterrés.

Sigles, NOTATIONS ET SYMBOLES

DDS : Direct Digital Synthesizer

GPR : Ground Penetrating RADAR

HBAR : High-overtone Bulk Acoustic Resonator SAR : Synthetic Aperture RADAR

SAW : Surface Acoustic Wave

\begin{tabular}{|c|l|c|}
\hline Symbole & Description & Unité \\
\hline$Q$ & facteur de qualité & sans \\
$k_{s}^{2}$ & couplage électromécanique & aucune \\
$f$ & fréquence de résonance & $\mathrm{Hz}$ \\
$\tau=Q /(\pi f)$ & constante de temps & $\mathrm{s}$ \\
$I L$ & pertes d'insertion & aucune \\
\hline
\end{tabular}


E3212 : capteurs acoustoélectriques radiofréquences modes d'interrogation

Radiofrequency acoustic passive wireless sensors interrogations strategies

par Jean-Michel Friedt - enseignant à l'Université de Franche-Comté, chercheur au département tempsfréqence de l'institut FEMTO-ST (Besançon), anciennement ingénieur chez SENSeOR - et Sylvain Ballandras - directeur général de frec|n|sys, une société du groupe SOITEC

\section{RÉFÉRENCES}

[1] Harry Stockman. Communication by means of reflected power. Proceedings of the IRE, 36(10) :1196-1204, 1948.

[2] Albert Glinsky. Theremin : ether music and espionage. University of Illinois Press, 2000.

[3] Xiaoju Yu, Min Liang, and Hao Xin. Performance evaluation of wideband microwave direction-of-arrival estimation using Luneburg lens. IEEE Antennas and Wireless Propagation Letters, 16 :2453-2456, 2017.

[4] J. Appelbaum, J. Horchert, and C. Stöcker. Shopping for spy gear : Catalog advertises NSA toolbox. Der Spiegel, Dec. 2013. leaksource.info/2013/12/30/nsas-antdivision-catalog-of-exploits-for-nearly-every-major-softwarehardware-firmware/

[5] Christopher T Allen, Kun Shi, and Richard G Plumb. The use of ground-penetrating radar with a cooperative target. IEEE transactions on geoscience and remote sensing, 36(5) :1821$1825,1998$.

[6] X Q. Bao, W. Burfhand an V.V. Varadan, and V.K. Varadan. SAW temperature sensor and remote reading system. In IEEE Ultrasonics Symposium, pages 583-585, 1987.

[7] W. Buff, F. Plath, 0. Schmeckebier, M. Rusko, T. Vandahl, H. Luck, F. Möller, and D.C. Malocha. Remote sensor system using passive saw sensors. In IEEE Ultrasonics Symposium, pages 585-588, 1994.

[8] A.Pohll, F.Seifert, L.Reind, G.Scholl, T. Ostertag, and W.Pietschl. Radio signals for saw id tags and sensors in strong electromagnetic interference. In IEEE Ultrasonics Symposium, pages 195-198, 1994.

[9] L. Reindl, G. Scholl, T. Ostertag, C.C.W. Ruppel, W.-E. Bulst, and F. Seifert. Saw devices as wireless passive sensors. In IEEE Ultrasonics Symposium, pages 363-367, 1996.

[10] W. Buff, M. Rusko, M. Goroll, J. Ehrenpfordt, and T. Vandahl. Universal pressure and temperature SAW sensor for wireless applications. In IEEE Ultrasonics Symposium, pages 359-362, 1997.

[11] A. Pohl and L. Reindl. Measurement of physical parameters of car tires using passive saw sensors. In Advanced Microsystems for Automotive Applications Conference, pages 250-262, 1998

[12] Victor P Plessky and Leonhard M Reindl. Review on SAW RFID tags. IEEE transactions on ultrasonics, ferroelectrics, and frequency control, 57(3), 2010.

[13] W. Buff, S. Klett, M. Rusko, J. Ehrenpfordt, and M. Goroli. Passive remote sensing for temperature and pressure using SAW resonator devices. IEEE Transactions on Ultrasonics, Ferroelectrics, and Frequency Control, 45(5) :1388-1392, september 1998

[14] V. Kalinin. Influence of receiver noise properties on resolution of passive wireless resonant saw sensors. In IEEE Ultrasonics Symposium, volume 3, pages 1452-1455, 2005.

[15] K.M. Lakin. Electrode resistance effects in interdigital transducers. IEEE Transactions on microwave theory and techniques, MTT-22(4) :418-424, April 1974.
[16] J.-M Friedt et al. High-overtone bulk acoustic resonator as passive sensor acting as buried cooperative target interrogated by ground penetrating RADAR. In IEEE International Frequency Control Symposium (IFCS), Prague, Czech Rep., 2013.

[17] C.S. Hartmann. A global saw id tag with large data capacity. In Proceedings of 2002 IEEE Ultrasonics Symposium, October 2002.

[18] C. S. Hartmann, P. Brown, and J. Bellamy. Design of global saw rfid tag devices. In Proceedings of the Second International Symposium on Acoustic Wave Devices for Future Mobile Communication Systems, March 2004.

[19] D.C. Malocha, M. Gallagher, B. Fisher, J. Humphries, D. Gallagher, and N. Kozlovski. A passive wireless multi-sensor SAW technology device and system perspectives. Sensors, $13: 5897-5922,2013$.

[20] C. Pfeffer, S. Scheiblhofer, R. Feger, and A. Stelzer. An s-fscw based multi-channel reader system for beamforming applications using surface acoustic wave sensors. Radioengineering, 20(4) :745-751, December 2011

[21] A. Stelzer, S. Scheiblhofer, S. Schuster, and M. Brandl. Multi reader/multi-tag saw rfid systems combining tagging, sensing, and ranging for industrial applications. In IFCS, 2008.

[22] CS Hartmann, P Brown, and J Bellamy. Design of global SAW RFID tag devices. In Proc. Second Int. Symp. on Acoustic Wave Devices for Future Mobile Commun. Syst., pages 1519, Chiba, Japan, 2004.

[23] Victor Plessky and Marc Lamothe. Ultra-wide-band SAW RFID/sensors. In European Frequency and Time Forum (EFTF), 2014, pages 16-23. IEEE, 2014.

[24] D. Puccio, D.C. Malocha, D.Gallagher, and J.Hines. SAW sensors using orthogonal frequency coding. In IEEE Frequency Control Symposium, pages 307-310, 2004.

[25] F. Lurz, T. Ostertag, B. Scheiner, R. Weigel, and A. Koelpin. Review : Reader architectures for wireless surface acoustic wave sensors. MPDI Sensors, 18(6) :1734, 2018.

[26] Weike Feng, Jean-Michel Friedt, Gwenhael Goavec-Merou, and Motoyuki Sato. Passive radar delay and angle of arrival measurements of multiple acoustic delay lines used as passive sensors. IEEE Sensors Journal, 19(2) :594-602, 2018.

[27] J.-M. Friedt, W. Feng, S. Chrétien, G. Goavec-Merou, and M. Sato. Passive radar for measuring passive sensors : direct signal interference suppression on FPGA using orthogonal matching pursuit and stochastic gradient descent. In Multimodal Sensing : Technologies and Applications, volume 11059, page 1105906 . International Society for Optics and Photonics, 2019.

[28] Melvin Paquit, Lilia Arapan, Weike Feng, and Jean-Michel Friedt. Long range passive radar interrogation of subsurface acoustic passive wireless sensors using terrestrial television signals. IEEE Sensors Journal, 2020.

[29] G. Goavec-Mérou, N. Chrétien, J.-M Friedt, P. Sandoz, G. Martin, M. Lenczner, and S.Ballandras. Fast contactless vibrating structure characterization using real time FPGAbased digital signal processing : demonstrations with a passive wireless acoustic delay line probe and vision. Rev. Sci. Instrum, 85(1) :015109, Jan. 2014.

[30] J.-M. Friedt, G. Martin, G. Goavec-Mérou, D. Rabus, S. Alzuaga, L. Arapan, M. Sagnard, and É. Carry. Acoustic transducers as passive cooperative targets for wireless sensing the sub-surface world : challenges of probing with ground penetrating RADAR. MDPI Sensors, 18(1) :246, Jan. 2018.

[31] J.-M Friedt, A. Saintenoy, S. Chrétien, T. Baron, E. Lebrasseur, T. Laroche, S. Ballandras, and M. Griselin. High-overtone bulk acoustic resonator as passive ground penetrating RADAR cooperative targets. J. Appl. Phys., 113(13) :134904, 2013. 
[32] T. Rétornaz, J.-M Friedt, S. Alzuaga, T. Baron, E. Lebrasseur, G. Martin, T. Laroche, S. Ballandras, M. Griselin, and J.-P. Simonnet. Piezoelectric radiofrequency transducers as passive buried sensors. Nondestructive Testing and Evaluation Special Issue on Civil Eng. Applications of GPR, 27(3) :209218, 2012.

[33] J.-M Friedt, T. Rétornaz, S. Alzuaga, T. Baron, G. Martin, T. Laroche, S. Ballandras, M. Griselin, and J.-P. Simonnet. Surface acoustic wave devices as passive buried sensors. Journal of Applied Physics, 109(3) :034905, 2011.

[34] M. Lamothe. Capteurs à ondes élastiques de surface à codage spectral Ultra Large Bande. $\mathrm{PhD}$ thesis, Universite de Franche Comté, Besançon, 122014.

[35] ETSI EN 302 065. Electromagnetic compatibility and radio spectrum matters (ERM); short range devices (SRD) using ultra wide band technology (UWB) for communication purposes. European harmonized standard EN, 10 :v1.2.1, 2010.

[36] G. Breed. A summary of FCC rules for ultra wideband communications. High Frequency Electronics, 4(1) :42-44, 2005

[37] G.L. Charvat. Small and Short-Range Radar Systems. CRC Press, 2014.

[38] J.-M Friedt. Gnuradio as a digital signal processing environment : application to acoustic wireless sensor measurement and time \& frequency analysis of periodic signals. In IFCS Prague, Czech Rep., 2013.

[39] J.L. Davis and A.P. Annan. Ground-penetrating radar for highresolution mapping of soil and rock stratigraphy. Geophys. Prospect., 37(5) :531-551, 1989.

[40] J.-M Friedt. Wideband measurement strategies : from radar to passive wireless sensors ... and how passive wireless sensors were/are used by intelligence agencies. In FOSDEM2016, Brussels, Belgium, 2016.

[41] J.-M Friedt. Passive cooperative targets for subsurface physical and chemical measurements : a systems perspective. IEEE Geoscience and Remote Sensing Letters, 14(6) :821-825, 2017.

[42] J.H. Kuypers, L.M. Reindl, S. Tanaka, and M. Esashi. Maximum accuracy evaluation scheme for wireless saw delayline sensors. IEEE Trans Ultrason Ferroelectr Freq Control, 55(7) :1640-52, Jul 2008.

[43] M. Hamsch, R. Hoffmann, W. Buff, M. Binhack, and S. Klett. An interrogation unit for passive wireless saw sensors based on fourier transform. IEEE transactions on ultrasonics, ferroelectrics, and frequency control, 51(11) :1449-1456, november 2004.

[44] J.-M Friedt, C. Droit, G. Martin, and S. Ballandras. A wireless interrogation system exploiting narrowband acoustic resonator for remote physical quantity measurement. Rev. Sci. Instrum., $81: 014701,2010$

[45] C. Droit, G. Martin, S. Ballandras, and J.-M Friedt. A frequency modulated wireless interrogation system exploiting narrowband acoustic resonator for remote physical quantity measurement. Rev. Sci. Instrum., 81 :056103, 2010.

[46] Weike Feng, Jean-Michel Friedt, Grigory Cherniak, and Motoyuki Sato. Passive bistatic radar using digital video broadcasting-terrestrial receivers as general-purpose softwaredefined radio receivers. Review of Scientific Instruments, 89(10) :104701, 2018.

[47] David Morgan. Surface acoustic wave filters : With applications to electronic communications and signal processing. Academic Press, 2010.

[48] J.-M Friedt, C. Droit, S. Ballandras, S. Alzuaga, G. Martin, and P. Sandoz. Remote vibration measurement : a wireless passive surface acoustic wave resonator fast probing strategy. Rev. Sci. Instrum., 83 :055001, May 2012.

[49] William Steichen and Sylvain Ballandras. Composants acoustiques utilisés pour le filtrage. Ed. Techniques Ingénieur, 2008.
[50] John R Vig. Quartz crystal resonators and oscillators. In IEEE Ultrasonics, Ferroelectrics and Frequency Control Symposium, tutorial, page 75, New Orleans, USA, 2000. https://www.am1.us/wp-content/uploads/Documents/ U11625_VIG-TUTORIAL.pdf

[51] J.F. Rosenbaum. Bulk Acoustic Wave Theory and Devices. Artech House, Boston, London, 1988.

[52] S. Ballandras and S. Alzuaga. One-port surface elastic wave resonator on high permittivity substrate, 2017. Patent US2017/0033840.

[53] J.-M. Friedt, G. Goavec-Merou, G. Martin, W. Feng, and M. Sato. Passive RADAR acoustic delay line sensor measurement : demonstration using a WiFi $(2.4 \mathrm{GHz})$ emitter and WAIC-band (4.3 ghz). In 6th IEEE International Conference on Wireless for Space and Extreme Environments (WiSEE), pages 54-61. IEEE, 2018

[54] L. Fagot-Revurat, S. Ballandras, J. Masson, and W. Steichen. Hybrid resonant structure to verify parameters of a tire, 2011.

[55] T-L Li, Z Wu, H Hu, and L Zheng. Pressure and temperature microsensor based on surface acoustic wave. Electronics letters, 45(6) :337-338, 2009.

[56] A. Pohl, R. Steindl, and L. Reindl. The "intelligent tire" utilizing passive SAW sensors - mesurement of tire friction. IEEE transactions on instrumentation and measurement, 48(6) :1041-1046, december 1999.

[57] K. Eun, K.-J. Lee, K.-K. Lee, S.-S. Yang, and S.-H. Choa. Highly sensitive surface acoustic wave strain sensor for the measurement of tire deformation. International Journal of Precision Engineering and Manufacturing, 17(6) :699-707, 2016.

[58] J-M Boccard, P Katus, R Renevier, LM Reindl, and J-M Friedt. Near-field interrogation of saw resonators on rotating machinery. Journal of Sensors and Sensor Systems, 2(2) :147156, 2013.

[59] Rory Stoney, Dermot Geraghty, and Garret E O'Donnell. Characterization of differentially measured strain using passive wireless surface acoustic wave (saw) strain sensors. IEEE Sensors Journal, 14(3) :722-728, 2014.

[60] J. Beckley, V. Kalinin, M. Lee, and K. Voliansky. Non-contact torque sensor based on saw resonators. In IEEE International Frequency Control Symposium and PDA Exhibition, pages 202-213, 2002.

[61] Jean-Louis Fanchon. Guide de mécanique. Nathan, 2001.

[62] JA Thiele and M Pereira Da Cunha. High temperature LGS SAW gas sensor. Sensors and Actuators B : Chemical, 113(2) :816-822, 2006.

[63] R Fachberger, G Bruckner, R Hauser, and L Reindl. Wireless saw based high-temperature measurement systems. In International Frequency Control Symposium and Exposition, 2006 IEEE, pages 358-367. IEEE, 2006.

[64] M Pereira Da Cunha, RJ Lad, T Moonlight, G Bernhardt, and DJ Frankel. High temperature stability of langasite surface acoustic wave devices. In Ultrasonics Symposium, 2008. IUS 2008. IEEE, pages 205-208. IEEE, 2008.

[65] DC Malocha, M Pereira Da Cunha, E Adler, RC Smythe, S Frederick, M Chou, R Helmbold, and YS Zhou. Recent measurements of material constants versus temperature for langatate, langanite and langasite. In Frequency Control Symposium and Exhibition, 2000. Proceedings of the 2000 IEEE/EIA International, pages 200-205. IEEE, 2000.

[66] M. Schulz, D. Richter, and H. Fritze. Material and resonator design dependant loss in langasite bulk acoustic wave resonators at high temperatures. In IEEE International Ultrasonics Symposium Proceedings, pages 1676-1679. IEEE, 2009. 


\section{POUR EN SAVOIR PLUS}

Conférences :

- IEEE Frequency Control Symposium IFCS, ieee-uffc.org/publications/internationalfrequency-control-symposium-proceedings/

- European Frequeny and Time Forum EFTF, www. eftf.org

Journaux :

- IEEE Sensors ieee-sensors.org/sensors-journal/

- IEEE Trans. Ultrasonics and Frequency Control ieee-uffc.org/publications/transactions-on-uffc/

- MDPI Sensors wWw.mdpi.com/journal/sensors 